\title{
Planar Waves of the Buffered Bistable System
}

\author{
Xiaohuan Wang ${ }^{1}$ and Guangying $\mathrm{Lv}^{1,2}$ \\ ${ }^{1}$ College of Mathematics and Information Science, Henan University, Kaifeng 475001, China \\ ${ }^{2}$ Institute of Contemporary Mathematics, Henan University, Kaifeng, Henan 475001, China
}

Correspondence should be addressed to Guangying Lv; gylvmaths@126.com

Received 12 June 2013; Accepted 9 October 2013

Academic Editor: Svatoslav Staněk

Copyright (C) 2013 X. Wang and G. Lv. This is an open access article distributed under the Creative Commons Attribution License, which permits unrestricted use, distribution, and reproduction in any medium, provided the original work is properly cited.

\begin{abstract}
This paper is concerned with the large time behavior of disturbed planar fronts in the buffered bistable system in $\mathbb{R}^{n}(n \geq 2)$. We first show that the large time behavior of the disturbed fronts can be approximated by that of the mean curvature flow with a drift term for all large time up to $t=+\infty$. And then we prove that the planar front is asymptotically stable in $L^{\infty}\left(\mathbb{R}^{n}\right)$ under ergodic perturbations, which include quasiperiodic and almost periodic ones as special cases.
\end{abstract}

\section{Introduction}

Traveling waves in excitable systems have been the subject of a vast number of mathematical studies for the past 50 years. The basic mathematical theory can be used to describe wave propagation in a wide array of biological and chemical systems. Recently, Tsai and Sneyd $[1,2]$ studied the following buffered system:

$$
\begin{gathered}
\frac{\partial u}{\partial t}=D \frac{\partial^{2} u}{\partial y^{2}}+f(u)+\sum_{i=1}^{N}\left[k_{-}^{i}\left(b_{0}^{i}-v_{i}\right)-k_{+}^{i} u v_{i}\right], \\
(y, t) \in \mathbb{R} \times(0, \infty), \\
\frac{\partial v_{i}}{\partial t}=D_{i} \frac{\partial^{2} v_{i}}{\partial y^{2}}+k_{-}^{i}\left(b_{0}^{i}-v_{i}\right)-k_{+}^{i} u v_{i}, \\
i=1, \ldots, N,
\end{gathered}
$$

where $D, D_{i}, k_{-}^{i}, k_{+}^{i}$ and $b_{0}^{i}$ are positive constants. We call system (1) the full buffering model. When $D_{i}=0$ $(i=1, \ldots, N)$, Tsai and Sneyd [1] proved the existence, uniqueness, and stability of traveling wave fronts of system (1). The existence, uniqueness, and stability of traveling wave fronts of system (1) with $D_{i} \neq 0$, were obtained by Tsai and Sneyd [2]. About the buffered system (3), see also [3, 4], for more details.
In this paper, we consider the large time behavior of the Cauchy problem to buffering model in $\mathbb{R}^{n}$ :

$$
\begin{gathered}
u_{t}=D \Delta u+f(u)+\sum_{i=1}^{N}\left[k_{-}^{i}\left(b_{0}^{i}-v_{i}\right)-k_{+}^{i} u v_{i}\right], \\
x \in \mathbb{R}^{n-1}, y \in \mathbb{R}, t>0, \\
v_{i t}=D_{i} \Delta v_{i}+k_{-}^{i}\left(b_{0}^{i}-v_{i}\right)-k_{+}^{i} u v_{i}, \\
i=1, \ldots, N,
\end{gathered}
$$

with initial value

$$
\begin{array}{r}
u(x, y, 0)=u_{0}(x, y), \\
x \in \mathbb{R}^{n-1}, y \in \mathbb{R}, \\
v_{i}(x, y, 0)=v_{i 0}(x, y), \\
x \in \mathbb{R}^{n-1}, y \in \mathbb{R},
\end{array}
$$

where $n \geq 2, \Delta=\partial^{2} / \partial x_{1}^{2}+\cdots+\partial^{2} / \partial x_{n-1}^{2}+\partial^{2} / \partial y^{2}$. Throughout this paper, we always assume that $D=D_{i}=1$. In other words, we consider a special case in the present paper and will study the general case in the further paper.

One of the most interesting and natural questions is the behavior of solutions $\left(u(x, y, t), v_{i}(x, y, t)\right)$ as $t \rightarrow+\infty$, in particular, the question about the stability of traveling wave 
fronts. Lv [5] studied the system (2) with $N=1$ and obtained the asymptotic stability of planar waves on $\mathbb{R}^{n}$, where $n \geq 2$. Under initial perturbation that decays at space infinity, they also proved that the perturbed solution converges to planar waves as $t \rightarrow \infty$. We remark that one can obtain the similar results to Theorems 1 and 3 when $N \geq 2$. For convenience, we only consider the case that $N=1$.

It is well known that (2) on $\mathbb{R}$ has a traveling wave solution with the form $\left(u(y, t), v_{i}(y, t)\right)=\left(\phi(y+c t), \psi_{i}(y+\right.$ $c t)$ ) for some constant $c \in \mathbb{R}$, connecting two equilibrium $\left(0, b_{0}^{i}\right)$ and $\left(1, k_{-}^{i} b_{0}^{i} /\left(k_{-}^{i}+k_{+}^{i}\right)\right)$, under the condition that $f$ is of bistable. Here " $f$ is bistable" is meant that $f^{\prime}(0)<0$ and $f^{\prime}(1)<0$, where $f(0)=f(1)=0$. A typical example is that $f(u)=u(u-a)(1-u)$, where $0<a<1$.

A traveling wave front of (2) is a monotone solution with the form $\left(u(y, t), v_{i}(y, t)\right)=\left(\phi(y+c t), \psi_{i}(y+c t)\right)(c$ is the wave speed), satisfying

$$
\begin{gathered}
D \phi^{\prime \prime}(\xi)-c \phi^{\prime}(\xi)+f(\phi(\xi)) \\
+\sum_{i=1}^{n}\left[k_{-}^{i}\left(b_{0}^{i}-\psi_{i}\right)-k_{+}^{i} \phi \psi_{i}\right]=0, \quad \xi \in \mathbb{R}, \\
D_{i} \psi_{i}^{\prime \prime}(\xi)-c \psi_{i}^{\prime}(\xi)+k_{-}^{i}\left(b_{0}^{i}-\psi_{i}\right)-k_{+}^{i} \phi \psi_{i}=0, \\
\quad \xi \in \mathbb{R}, \\
\phi^{\prime}(\xi)>0, \psi_{i}^{\prime}(\xi)<0, \\
\lim _{\xi \rightarrow-\infty}\left(\phi(\xi), \psi_{i}(\xi)\right)=\left(0, b_{0}^{i}\right), \\
\lim _{\xi \rightarrow \infty}\left(\phi(\xi), \psi_{i}(\xi)\right)=\left(1, b_{2}^{i}\right),
\end{gathered}
$$

where $\xi=y+c t,{ }^{\prime}=d / d \xi$ and $b_{2}^{i}=k_{-}^{i} b_{0}^{i} /\left(k_{-}^{i}+k_{+}^{i}\right)$.

The function $\left(\phi(y+c t), \psi_{i}(y+c t)\right)$ is also a traveling wave front for system (2) with $n \geq 2$. We call it planar front. Its translation $\left(\phi(y+c t+\xi), \psi_{i}(y+c t+\xi)\right)$, where $\xi$ is any constant, is also called a planar front. Note that the equations in (2) from second to the last have the same properties; without loss of generality, we only consider the case that $N=$ 1 , and we write $v_{i}$ as $v$, that is,

$$
\begin{gathered}
u_{t}=\Delta u+f(u)+k_{1}\left(b_{0}-v\right)-k_{2} u v, \\
x \in \mathbb{R}^{n-1}, y \in \mathbb{R}, t>0, \\
v_{t}=\Delta v+k_{1}\left(b_{0}-v\right)-k_{2} u v, \\
x \in \mathbb{R}^{n-1}, y \in \mathbb{R}, t>0,
\end{gathered}
$$

with initial value

$$
\begin{array}{ll}
u(x, y, 0)=u_{0}(x, y), & x \in \mathbb{R}^{n-1}, y \in \mathbb{R}, \\
v(x, y, 0)=v_{0}(x, y), & x \in \mathbb{R}^{n-1}, y \in \mathbb{R} .
\end{array}
$$

Let $u^{*}=u$ and $v^{*}=b_{0}-v$, then (5) becomes (for simplicity, we omit the symbol $*$ )

$$
\begin{gathered}
u_{t}=\Delta u+f(u)+k_{1} v-k_{2} u\left(b_{0}-v\right), \\
x \in \mathbb{R}^{n-1}, y \in \mathbb{R}, t>0, \\
v_{t}=\Delta v-k_{1} v+k_{2} u\left(b_{0}-v\right), \\
x \in \mathbb{R}^{n-1}, y \in \mathbb{R}, t>0 .
\end{gathered}
$$

Obviously, system (7) is a cooperative model. The problem corresponding to traveling wave front $(u(x, t), v(x, t))=$ $(\phi(\xi), \psi(\xi))$ of system $(7)$, connecting $(0,0)$ and $\left(1, b_{0}-b_{2}\right)$, is

$$
\begin{gathered}
\phi^{\prime \prime}-c \phi^{\prime}+f(\phi)+k_{1} \psi-k_{2} \phi\left(b_{0}-\psi\right)=0, \\
\psi^{\prime \prime}-c \psi^{\prime}-k_{1} \psi+k_{2} \phi\left(b_{0}-\psi\right)=0, \\
\phi^{\prime}>0, \\
\psi^{\prime}>0, \\
\lim _{\xi \rightarrow-\infty}(\phi, \psi)=(0,0), \\
\lim _{\xi \rightarrow \infty}(\phi, \psi)=\left(1, b_{0}-b_{2}\right) .
\end{gathered}
$$

Recently, Matano et al. [6] considered the following Allen-Cahn equation:

$$
\begin{gathered}
u_{t}=\Delta u+f(u), \quad x \in \mathbb{R}^{n-1}, y \in \mathbb{R}, t>0, \\
u(x, y, 0)=u_{0}(x, y), \quad x \in \mathbb{R}^{n-1}, y \in \mathbb{R} .
\end{gathered}
$$

Under the condition that $f$ is of the bistable type, they obtained the stability of planar fronts under any-possibly large-initial perturbations and almost periodic perturbations. When $f$ is monostable type, the stability of planar waves for (9) was obtained by Lv and Wang [7]. About the stability of planar wave, also see [8-10]. Just recently, Matano and Nara [11] reconsidered the Cauchy problem (9) under the condition that $f$ is bistable type. They proved that the planar front is asymptotically stable in $L^{\infty}\left(\mathbb{R}^{n}\right)$ under spatially ergodic perturbation and that the large time behavior of the disturbed planar front can be approximated by that of the mean curvature flow with a drift term for all large time up to $+\infty$. Lv [12] studied the Cauchy problem (9) under that $f$ is monostable type and obtained that the planar front is asymptotically stable in $L^{\infty}\left(\mathbb{R}^{n}\right)$ under spatially ergodic perturbation.

Our work has been inspired by [11]; in this paper, we will consider the stability of planar fronts under spatially ergodic perturbation. Let us now state our main results. 
Theorem 1 (large time behavior). Let $n \geq 2$. Let $(u(x$, $y, t), v(x, y, t))$ be a solution problem (7) with (6) whose initial value $\left(u_{0}(x, y), v_{0}(x, y)\right)$ satisfies

$$
\begin{aligned}
&(0,0) \leq\left(u_{0}(x, y), v_{0}(x, y)\right) \leq\left(1, b_{0}-b_{2}\right), \\
& \limsup _{y \rightarrow-\infty} \sup _{x \in \mathbb{R}^{n-1}} u_{0}(x, y)<\delta, \\
& \liminf _{y \rightarrow+\infty} \inf _{x \in \mathbb{R}^{n-1}} u_{0}(x, y)>1-\delta, \\
& \limsup _{y \rightarrow-\infty} \sup _{x \in \mathbb{R}^{n-1}} v_{0}(x, y)<k_{0} \delta, \\
& \liminf _{y \rightarrow+\infty} \inf _{x \in \mathbb{R}^{n-1}} v_{0}(x, y)>b_{0}-b_{2}-k_{0} \delta,
\end{aligned}
$$

where $\delta \in\left(0, \delta_{0}\right]$ and $k_{0}>0$ are defined as in Lemma 4. Then there exist a constant $T>0$ and a smooth function $\gamma(x, t)$ such that

(i) for each $t \in[T,+\infty)$ and $x \in \mathbb{R}^{n-1}$, one has ( $u(x$, $y, t), v(x, y, t))=(\phi(0), \psi(0))$ if and only if $y=$ $\gamma(x, t)$;

(ii) it holds that

$$
\begin{aligned}
& \lim _{t \rightarrow \infty} \sup _{(x, y) \in \mathbb{R}^{n}}|u(x, y, t)-\phi(y-\gamma(x, t))|=0, \\
& \lim _{t \rightarrow \infty} \sup _{(x, y) \in \mathbb{R}^{n}}|v(x, y, t)-\psi(y-\gamma(x, t))|=0 ;
\end{aligned}
$$

(iii) for any $\varepsilon>0$, there exists $\tau_{\varepsilon} \in[T, \infty)$ such that the solution $U(x, t)$ of the problem

$$
\begin{gathered}
\frac{U_{t}}{\sqrt{1+\left|\nabla_{x} U\right|^{2}}}=\operatorname{div}\left(\frac{\nabla_{x} U}{\sqrt{1+\left|\nabla_{x} U\right|^{2}}}\right)-c, \\
x \in \mathbb{R}^{n-1}, t>0, \\
U(x, 0)=\gamma\left(x, \tau_{\varepsilon}\right), \quad x \in \mathbb{R}^{n-1}
\end{gathered}
$$

satisfies

$$
\sup _{x \in \mathbb{R}^{n-1}, t \geq \tau_{\varepsilon}}\left|\gamma(x, t)-U\left(x, t-\tau_{\varepsilon}\right)\right| \leq \varepsilon,
$$

where $\nabla_{x}$ denotes the $(n-1)$-dimensional gradient.

The statement (ii) of Theorem 1 implies that the solution $(u(x, y, t), v(x, y, t))$ behaves like the function $(\phi(y-$ $\gamma(x, t)), \psi(y-\gamma(x, t)))$ for large $t$; thus the large time behavior of the solution $(u(x, y, t), v(x, y, t))$ is basically determined by the position of the $(\phi(0), \psi(0))$-level set $(\gamma(x, t), \gamma(x, t))$. The last statement shows that the behavior of $\gamma(x, t)$ can be approximated by the solution $U(x, t)$ of the mean curvature flow on $\mathbb{R}^{n-1}$ with a drift term $c$. Comparing the above theorem with Theorem 1.1 in [5], it is to see that we delete the assumptions that initial perturbations decay to zero as $|x|+$ $|y| \rightarrow \infty$, and thus the result in this paper is better than that of [5].

In order to obtain the stability of planar wave, we need the following definition.
Definition 2 (unique ergodicity in the $x$-direction). A bounded uniformly continuous function $p(x, y): \mathbb{R}^{n-1} \times \mathbb{R} \rightarrow$ $\mathbb{R}$ is called uniquely ergodic in the $x$-direction if there exists a unique probability measure on $\mathbb{H}_{p}$, that is, $\sigma_{a}$-invariant for any $a \in \mathbb{R}^{n-1}$,

$$
\begin{gathered}
\mathbb{H}_{p}:=\overline{\left\{\sigma_{a} g \mid a \in \mathbb{R}^{n-1}\right\}} L_{\mathrm{loc}}^{\infty}\left(\mathbb{R}^{n-1}\right), \\
\left(\sigma_{a} p\right)(x)=p(x+a),
\end{gathered}
$$

and $\bar{A}^{X}$ stands for the closure of a set $A$ in the $X$-topology.

Theorem 3. In addition to the assumptions of Theorem 1, assume further that $u_{0}(x, y)$ and $v_{0}(x, y)$ are uniquely ergodic in the $x$-direction. Then there exist constants $\mu_{1}, \mu_{2} \in \mathbb{R}$ such that

$$
\begin{aligned}
& \lim _{t \rightarrow \infty} \sup _{(x, y) \in \mathbb{R}^{n}}\left|u(x, y, t)-\phi\left(y+c t+\mu_{1}\right)\right|=0, \\
& \lim _{t \rightarrow \infty} \sup _{(x, y) \in \mathbb{R}^{n}}\left|v(x, y, t)-\psi\left(y+c t+\mu_{2}\right)\right|=0 .
\end{aligned}
$$

From [11], we see that

$$
\mathbb{P} \subset \mathbb{Q P} \subset \mathbb{A P} \subset \mathbb{S E} \subset \mathbb{U} \mathbb{E},
$$

where $\mathbb{P}, \mathbb{Q P}, \mathbb{A P}, \mathbb{S} \mathbb{E}, \mathbb{E}$ denote, respectively, the sets of periodic functions, quasiperiodic functions, almost periodic functions, strictly ergodic functions, and uniquely ergodic functions. Hence, the above Theorem 3 is a general result.

The rest of this paper is organized as follows. In Section 2, some known results and related problem are considered. Section 3 is concerned with the proofs of Theorems 1 and 3.

\section{Some Known Results and Related Problem}

In this section, we first study the one-dimensional problem (7) in a moving frame, that is,

$$
\begin{gathered}
u_{t}=u_{z z}-c u_{z}+f(u)+k_{1} v-k_{2} u\left(b_{0}-v\right), \\
z \in \mathbb{R}, t>0, \\
v_{t}=v_{z z}-c v_{z}-k_{1} v+k_{2} u\left(b_{0}-v\right), \\
z \in \mathbb{R}, t>0,
\end{gathered}
$$

where $z=y+c t$.

The traveling wave solutions of (17) have been studied by many authors; see $[1,2]$, for more details. Tsai and Sneyd $[1,2]$ obtained the existence, uniqueness, and stability of traveling wave fronts of system (17). They obtained the following lemmas; see Lemma 6.1 in [2] and Lemma 3.5 in [5].

Lemma 4. Assume that $f$ is bistable and $(\phi(z), \psi(z))$ is a traveling wave solution of (17). Then there exist $\delta_{0}, \beta, k_{0}>0$ and $\sigma \geq 1$ such that, for any $\delta \in\left(0, \delta_{0}\right]$, the function defined by

$$
\begin{gathered}
u^{+}(x, z, t)=\phi\left(z+\sigma \delta\left(1-e^{-\beta t}\right)\right)+\delta e^{-\beta t}, \\
v^{+}(x, z, t)=\psi\left(z+\sigma \delta\left(1-e^{-\beta t}\right)\right)+k_{0} \delta e^{-\beta t}
\end{gathered}
$$


is a subsolution. More precisely, it satisfies the following inequality:

$$
\begin{aligned}
N\left[u^{+}\right]:= & u_{t}^{+}-\Delta u^{+}+c u_{z}^{+}-f\left(u^{+}\right) \\
& -k_{1} v^{+}+k_{2} u^{+}\left(b_{0}-v^{+}\right) \geq 0, \\
N\left[v^{+}\right]:= & v_{t}^{+}-\Delta v^{+}+c v_{z}^{+}+k_{1} v^{+} \\
& -k_{2} u^{+}\left(b_{0}-v^{+}\right) \geq 0 .
\end{aligned}
$$

Lemma 5. Assume that $f$ is bistable and $(\phi(z), \psi(z))$ is a traveling wave solution of (17). Then there exist $\delta_{0}, \beta, k_{0}>0$ and $\sigma \geq 1$ such that, for any $\delta \in\left(0, \delta_{0}\right]$, the function defined by

$$
\begin{gathered}
u^{-}(x, z, t)=\phi\left(z-\sigma \delta\left(1-e^{-\beta t}\right)\right)-\delta e^{-\beta t}, \\
v^{-}(x, z, t)=\psi\left(z-\sigma \delta\left(1-e^{-\beta t}\right)\right)-k_{0} \delta e^{-\beta t}
\end{gathered}
$$

is a subsolution. More precisely, it satisfies the following inequality:

$$
\begin{gathered}
N\left[u^{-}\right]:=u_{t}^{-}-\Delta u^{-}+c u_{z}^{-}-f\left(u^{-}\right) \\
-k_{1} v^{-}+k_{2} u^{-}\left(b_{0}-v^{-}\right) \leq 0, \\
N\left[v^{-}\right]:=v_{t}^{-}-\Delta v^{-}+c v_{z}^{-}+k_{1} v^{-}-k_{2} u^{-}\left(b_{0}-v^{-}\right) \leq 0 .
\end{gathered}
$$

The proof of the above two lemmas is similar to that of Lemmas 3.5 and 3.6 in [5], and we omit them here. Now we consider the following problem:

$$
\begin{gathered}
\frac{U_{t}}{\sqrt{1+\left|\nabla_{x} U\right|^{2}}}=\operatorname{div}\left(\frac{\nabla_{x} U}{\sqrt{1+\left|\nabla_{x} U\right|^{2}}}\right)-c, \\
x \in \mathbb{R}^{m}, t>0, \\
U(x, 0)=U_{0}(x), \quad x \in \mathbb{R}^{m} .
\end{gathered}
$$

Lemma 6 (see [11]). Let $m \geq 1$. Let $U(x, t)$ be a solution to the problem (22) whose initial value $U_{0}(x)$ is bounded, Lipschitz continuous, and uniquely ergodic on $\mathbb{R}^{m}$. Then there exists a constant $\mu \in \mathbb{R}$ such that

$$
\lim _{t \rightarrow \infty} \sup _{x \in \mathbb{R}^{m}}|U(x, t)+c t+\mu|=0 .
$$

Lemma 7 (see [11]). Let $U(x, t ; \varphi)$ and $V(x, t ; \varphi)$ denote the solutions of the following equations:

$$
\begin{array}{r}
\frac{U_{t}}{\sqrt{1+\left|\nabla_{x} U\right|^{2}}}=\operatorname{div}\left(\frac{\nabla_{x} U}{\sqrt{1+\left|\nabla_{x} U\right|^{2}}}\right)-c, \\
x \in \mathbb{R}^{m}, t>0, \\
V_{t}=\Delta_{x} V-\frac{c}{2}\left|\nabla_{x} V\right|^{2}-c, \quad x \in \mathbb{R}^{m}, t>0,
\end{array}
$$

under the initial conditions $U(\cdot, 0)=V(\cdot, 0)=\varphi \epsilon$ $W^{2, \infty}\left(\mathbb{R}^{m}\right)$. Then, for any constant $\varepsilon>0$, there exists a constant $\delta>0$ such that if $\left\|\nabla_{x} \varphi\right\|_{W^{1, \infty}} \leq \delta$, it holds that

$$
\sup _{x \in \mathbb{R}^{m}}|U(x, t ; \varphi)-V(x, t ; \varphi)| \leq \varepsilon \quad \forall t>0 .
$$

The following lemma is a little different from Lemma 3.10 in [11], but the proof is similar.

Lemma 8. Let $V(x, t)$ be a solution to the problem

$$
\begin{gathered}
V_{t}=\Delta_{x} V-\frac{c}{2}\left|\nabla_{x} V\right|^{2}-c, \quad x \in \mathbb{R}^{n-1}, t>0, \\
V(x, 0)=V_{0}(x), \quad x \in \mathbb{R}^{n-1} .
\end{gathered}
$$

Then the following estimates hold:

$$
\begin{gathered}
\sup _{x \in \mathbb{R}^{n-1}}\left|V_{x_{i}}(x, t)\right| \leq \min \left\{C_{0} t^{-1 / 2}, C_{1}\right\}, \\
\sup _{x \in \mathbb{R}^{n-1}}\left|V_{x_{i} x_{j}}(x, t)\right| \leq \min \left\{C_{0} t^{-1}, C_{2}\right\}, \\
\sup _{x \in \mathbb{R}^{n-1}}\left|V_{x_{i} x_{j} x_{k}}(x, t)\right| \leq C_{3}(1+t)^{-3 / 2}, \\
\sup _{x \in \mathbb{R}^{n-1}}\left|V_{x_{i} t}(x, t)\right| \leq C_{4}(1+t)^{-3 / 2},
\end{gathered}
$$

for each $1 \leq i, j, k \leq n-1$, where $C_{0}, C_{1}, C_{2}, C_{3}$, and $C_{4}$ are positive constants such that

(i) $C_{0}$ depends only on $c$ and $\left\|V_{0}\right\|_{L^{\infty}}$;

(ii) $C_{1}$ depends only on $c,\left\|V_{0}\right\|_{L^{\infty}}$, and $\left\|\nabla_{x} V_{0}\right\|_{L^{\infty}}$ and satisfies

$$
C_{1} \longrightarrow 0 \quad \text { as }\left\|\nabla_{x} V_{0}\right\|_{L^{\infty}} \longrightarrow 0
$$

(iii) $C_{2}$ depends only on $c,\left\|V_{0}\right\|_{L^{\infty}}$, and $\left\|\nabla_{x} V_{0}\right\|_{W^{1, \infty}}$ and satisfies

$$
C_{2} \longrightarrow 0 \text { as }\left\|\nabla_{x} V_{0}\right\|_{W^{1, \infty}} \longrightarrow 0 ;
$$

(iv) $C_{3}$ and $C_{4}$ depend only on $c$ and $\left\|V_{0}\right\|_{W^{3, \infty}}$.

\section{Proofs of Theorems 1 and 3}

In this section, we consider problem (7) in $\mathbb{R}^{n}$ and prove our main results. Firstly, we give rough upper and lower bounds for the solution at large time, then introduce the notion of $\omega$-limit points of the solution and study basic properties of $(\phi(0), \psi(0))$-level surface of the solution. Lastly, we construct a fine set of supersolutions and subsolutions and give the proofs of the main theorems.

We will express solutions $(u(x, y, t), v(x, y, t))$ of $(1)$ in a moving frame, and thus the planar waves can be viewed as stationary states. Letting

$$
\begin{aligned}
w(x, z, t) & =u(x, y, t), \omega(x, z, t) \\
& =v(x, y, t), \quad z=y+c t,
\end{aligned}
$$


problem (7) with (6) is rewritten as (we write $(w(x$, $z, t), \omega(x, z, t))$ as $(u(x, z, t), v(x, z, t))$ for simplicity)

$$
\begin{gathered}
u_{t}=\Delta u-c u_{z}+f(u)+k_{1} v-k_{2} u\left(b_{0}-v\right), \\
x \in \mathbb{R}^{n-1}, \quad z \in \mathbb{R}, t>0, \\
v_{t}=\Delta v-c v_{z}-k_{1} v+k_{2} u\left(b_{0}-v\right), \\
x \in \mathbb{R}^{n-1}, \quad z \in \mathbb{R}, \quad t>0, \\
u(x, z, 0)=u_{0}(x, z), \quad x \in \mathbb{R}^{n-1}, \quad z \in \mathbb{R}, \\
v(x, z, 0)=v_{0}(x, z), \quad x \in \mathbb{R}^{n-1}, \quad z \in \mathbb{R},
\end{gathered}
$$

where $\Delta=\partial^{2} / \partial x_{1}^{2}+\cdots+\partial^{2} / \partial x_{n-1}^{2}+\partial^{2} / \partial z^{2}$. Throughout this section, we always assume that the initial value $\left(u_{0}, v_{0}\right)$ satisfies $(0,0) \leq\left(u_{0}(x, z), v_{0}(x, z)\right) \leq$ $\left(1, b_{0}-b_{2}\right)$ and

$$
\begin{gathered}
\limsup _{z \rightarrow-\infty} \sup _{x \in \mathbb{R}^{n-1}} u_{0}(x, z)<\delta, \\
\liminf _{z \rightarrow+\infty} \inf _{x \in \mathbb{R}^{n-1}} u_{0}(x, z)>1-\delta, \\
\limsup _{z \rightarrow-\infty} \sup _{x \in \mathbb{R}^{n-1}} v_{0}(x, z)<k_{0} \delta, \\
\liminf _{z \rightarrow+\infty} \inf _{x \in \mathbb{R}^{n-1}} v_{0}(x, z)>b_{0}-b_{2}-k_{0} \delta,
\end{gathered}
$$

where $\delta$ and $k_{0}$ are defined in Theorem 1 . We first consider upper and lower bounds of the solution of (31) at large time.

Lemma 9. Let $(u(x, z, t), v(x, z, t))$ be a solution of (31). Then there exists constant $z_{*}, z^{*} \in \mathbb{R}$ such that

$\lim _{t \rightarrow \infty} \inf _{x \in \mathbb{R}^{n-1}}(u(x, z, t), v(x, z, t)) \geq\left(\phi\left(z-z^{*}\right), \psi\left(z-z^{*}\right)\right)$,

uniformly in $z \in \mathbb{R}$,

$\lim _{t \rightarrow \infty} \sup _{x \in \mathbb{R}^{n-1}}(u(x, z, t), v(x, z, t)) \leq\left(\phi\left(z-z_{*}\right), \psi\left(z-z_{*}\right)\right)$,

uniformly in $z \in \mathbb{R}$.

Proof. We only show the upper bound of $(u(x, z, t), v(x, z, t))$, since the other is similar. Let $\left(u^{+}, v^{+}\right)$be as in Lemma 4. Then it suffices to show that there exist constants $T>0$ and $z_{0} \in \mathbb{R}$ such that

$$
\begin{gathered}
u(x, z, T) \leq \phi\left(z-z_{0}\right)+\delta_{1}=u^{+}\left(z-z_{0}, 0\right), \\
v(x, z, T) \leq \psi\left(z-z_{0}\right)+\delta_{2}=v^{+}\left(z-z_{0}, 0\right) .
\end{gathered}
$$

Indeed, the comparison principle and (34)-(35) give $(u(x, z, t), v(x, z, t)) \leq\left(u^{+}\left(z-z_{0}, t-T\right), v^{+}\left(z-z_{0}, t-T\right)\right)$ for $t \geq T$, which yield upper bound by letting $t \rightarrow \infty$.

Since $(0,0) \leq\left(u_{0}(x, z), v_{0}(x, z)\right) \leq\left(1, b_{0}-b_{2}\right)$, we see from the comparison principle that

$$
\begin{array}{r}
(0,0) \leq(u(x, z, t), v(x, z, t)) \leq\left(1, b_{0}-b_{2}\right), \\
(x, z) \in \mathbb{R}^{n}, t>0 .
\end{array}
$$

Note that $\lim _{z \rightarrow \infty}(\phi(z), \psi(z))=\left(1, b_{0}-b_{2}\right)$, there exists a constant $z_{1} \in \mathbb{R}$ such that, for each $T>0$ and $z \geq z_{1}$,

$$
\begin{aligned}
& u(x, z, t) \leq \phi(z)+\delta_{1}=u^{+}(z, 0), \\
& v(x, z, t) \leq \psi(z)+\delta_{2}=v^{+}(z, 0) .
\end{aligned}
$$

Next, we show that

$$
\begin{aligned}
& \left(\limsup _{z \rightarrow-\infty} \sup _{x \in \mathbb{R}^{n-1}} u(x, z, T), \limsup _{z \rightarrow-\infty} \sup _{x \in \mathbb{R}^{n-1}} v(x, z, T)\right) \\
& \quad<\left(\delta_{1}, \delta_{2}\right),
\end{aligned}
$$

for each $T>0$. For this purpose, choose positive constants $\beta_{1}, \beta_{2}$, and $M$ such that

$$
\begin{gathered}
\limsup _{z \rightarrow-\infty} \sup _{x \in \mathbb{R}^{n-1}} u_{0}(x, z)<\beta_{1}<\delta, \\
\limsup _{z \rightarrow-\infty} \sup _{x \in \mathbb{R}^{n-1}} v_{0}(x, z)<\beta_{2}<k_{0} \delta
\end{gathered}
$$

and that

$$
\begin{array}{r}
\left(u_{0}(x, z), v_{0}(x, z)\right) \leq\left(\beta_{1}+M e^{c z}, \beta_{2}+M e^{c z}\right), \\
(x, z) \in \mathbb{R}^{n} .
\end{array}
$$

Then the functions

$$
\begin{aligned}
& (w(x, z, t), \omega(x, z, t)) \\
& \quad=\left(\min \left\{\beta_{1}+M e^{c(z+a t)}, 1\right\}, \min \left\{\beta_{2}+M e^{c(z+a t)}, b_{0}-b_{2}\right\}\right)
\end{aligned}
$$

are a supersolution of (31) if $a>0$ is chosen sufficiently large. Hence,

$$
(u(x, z, T), v(x, z, T)) \leq(w(x, z, T), \omega(x, z, T)) .
$$

This proves (38). Combining (37) and (38), it is easy to see that (34)-(35) hold. This completes the proof.

Now, we introduce the notion of $\omega$-limit points of the solution $(u(x, z, t), v(x, z, t))$ of (31), where we consider a sequence both in $x$ and $t$. Then we show that any $\omega$-limit point is a planar wave under the assumption (34).

Definition 10. A vector-valued function $(w(x, z, t), \omega(x, z, t))$ defined on $\mathbb{R}^{n-1} \times \mathbb{R} \times \mathbb{R}$ is called a $\omega$-limit point of the solution $(u(x, z, t), v(x, z, t))$ of (31) if there exists a sequence $\left\{\left(x_{i}, t_{i}\right)\right\}$ such that $0<t_{1}<t_{2}<\cdots \rightarrow \infty$ and that

$$
\begin{aligned}
& \left(u\left(x+x_{i}, z, t+t_{i}\right), v\left(x+x_{i}, z, t+t_{i}\right)\right) \\
& \quad \longrightarrow(w(x, z, t), \omega(x, z, t)) \quad \text { as } i \longrightarrow \infty \text { in } C_{\text {loc }}^{2,1}\left(\mathbb{R}^{n} \times \mathbb{R}\right) .
\end{aligned}
$$

The following remark tells us how to construct $\omega$-limit point, which is similar to Remark 4.4 in [11]. 
Remark 11. Let $\mathbf{U}(x, z, t):=(u(x, z, t), v(x, z, t))$ be a solution of (31). Then for any sequence $\left\{\left(x_{i}, t_{i}\right)\right\}$ with $0<t_{1}<$ $t_{2}<\cdots \rightarrow \infty$, there exist a subsequence $\left\{\left(x_{i}^{\prime}, t_{i}^{\prime}\right)\right\}$ and a $\omega$-limit point $\mathbf{W}(x, z, t):=(w(x, z, t), \omega(x, z, t))$ of $\mathbf{U}(x, z$, t) such that

$$
\begin{array}{r}
\mathbf{U}\left(x+x_{i}^{\prime}, z, t+t_{i}^{\prime}\right) \longrightarrow \mathbf{W}(x, z, t) \\
\text { as } i \longrightarrow \infty \text { in } C_{\text {loc }}^{2,1}\left(\mathbb{R}^{n} \times \mathbb{R}\right)
\end{array}
$$

Indeed, by the assumption in Theorem 1, it is easy to see that $\mathbf{U}(x, z, t)$ is bounded. Then by $L^{p}$-estimates and Schauder's estimate (see $[13,14])$, the solution $\mathbf{U}(x, z$, t) belongs to $C^{2+\alpha, 1+\alpha / 2}\left(\mathbb{R}^{n} \times[\delta, T]\right)$ for any $0<\delta<T$. Furthermore,

$$
\|\mathbf{U}(x, z, t)\|_{C^{2+\alpha, 1+\alpha / 2}\left(\mathbb{R}^{n} \times[\delta, T]\right)} \leq C,
$$

where $C>0$ is a constant independent of $T>0$. Let $\left\{Q_{k}\right\}_{k=1,2, \ldots}$ be a sequence of compact subsets of $\mathbb{R}^{n} \times \mathbb{R}$ satisfying

$$
Q_{1} \subset Q_{2} \subset \cdots, \quad \lim _{k \rightarrow \infty} Q_{k}=\mathbb{R}^{n} \times \mathbb{R} .
$$

Then, for each $k$, the sequence of functions $\{\mathbf{U}(x, z, t)(x+$ $\left.\left.x_{i}, z, t+t_{i}\right)\right\}_{i=1,2, \ldots}$ is defined on $Q_{k}$ for all large $i$ and the restrictions of these functions onto $Q_{k}$ are relatively compact in $C^{2,1}\left(Q_{k}\right)$ by virtue of the estimate (45). By using diagonal argument, we can choose a subsequence $\left\{\left(x_{i}^{\prime}, t_{i}^{\prime}\right)\right\}$ and a function $w(x, z, t)$ defined on $\mathbb{R}^{n} \times \mathbb{R}$ such that, for any $k \geq$ 1 , it holds that

$$
\lim _{i \rightarrow \infty}\left\|\mathbf{U}\left(x+x_{i}^{\prime}, z, t+t_{i}^{\prime}\right)-\mathbf{W}(x, z, t)\right\|_{C^{2,1}\left(Q_{k}\right)}=0,
$$

which means $\mathbf{U}\left(x+x_{i}^{\prime}, z, t+t_{i}^{\prime}\right) \rightarrow \mathbf{W}(x, z, t)$ as $i \rightarrow$ $\infty$ in $C_{\text {loc }}^{2,1}\left(Q_{k}\right)$.

In order to prove that any $\omega$-limit point is a planar wave, the following Lemma is needed.

Lemma 12. Let $(u(x, z, t), v(x, z, t))$ be a vector-valued function that is defined on $\mathbb{R}^{n-1} \times \mathbb{R} \times \mathbb{R}$ and satisfies

$$
\begin{gathered}
u_{t}=\Delta u-c u_{z}+f(u)+k_{1} v-k_{2} u\left(b_{0}-v\right), \\
(x, z) \in \mathbb{R}^{n}, t \in \mathbb{R}, \\
v_{t}=\Delta v-c v_{z}-k_{1} v+k_{2} u\left(b_{0}-v\right), \\
(x, z) \in \mathbb{R}^{n}, t \in \mathbb{R} .
\end{gathered}
$$

Assume further that there exist two constants $z_{*}, z^{*} \in$ $\mathbb{R}$ such that

$$
\begin{aligned}
& \left(\phi\left(z-z^{*}\right), \psi\left(z-z^{*}\right)\right) \\
& \quad \leq(u(x, z, t), v(x, z, t)) \\
& \quad \leq\left(\phi\left(z-z_{*}\right), \psi\left(z-z_{*}\right)\right), \quad(x, z) \in \mathbb{R}^{n}, t \in \mathbb{R} .
\end{aligned}
$$

Then there exists a constant $z_{0} \in\left[z_{*}, z^{*}\right]$ such that

$$
\begin{array}{r}
u(x, z, t)=\phi\left(z-z_{0}\right), \\
v(x, z, t)=\psi\left(z-z_{0}\right), \\
(x, z) \in \mathbb{R}^{n}, t \in \mathbb{R} .
\end{array}
$$

Proof. Define

$$
\begin{array}{r}
u^{s}(x, z, t)=u(x+y, z+s, t+T), \\
y \in \mathbb{R}^{n-1}, s, T \in \mathbb{R}, \\
v^{s}(x, z, t)=v(x+y, z+s, t+T), \\
y \in \mathbb{R}^{n-1}, s, T \in \mathbb{R} .
\end{array}
$$

Then,

$$
\begin{aligned}
(\phi(z & \left.\left.+s-z^{*}\right), \psi\left(z+s-z^{*}\right)\right) \\
& \leq\left(u^{s}(x, z, t), v^{s}(x, z, t)\right) \\
& \leq\left(\phi\left(z+s-z_{*}\right), \psi\left(z+s-z_{*}\right)\right) .
\end{aligned}
$$

It follows from the monotonicity of $\phi$ and $\psi$ that there exists $s \in \mathbb{R}$ satisfying

$$
\begin{aligned}
& u^{s}(x, z, t) \geq u(x, z, t), \\
& v^{s}(x, z, t) \geq v(x, z, t) .
\end{aligned}
$$

Indeed, let $s=z^{*}-z_{*}$, then $\left(u^{s}(x, z, t), v^{s}(x, z, t)\right) \geq$ $(u(x, z, t), v(x, z, t))$. Now, let

$$
s_{*}=\inf \left\{s \in \mathbb{R},\left(u^{s^{\prime}}, v^{s^{\prime}}\right) \geq(u, v) \text {, in } \mathbb{R}^{n} \times \mathbb{R} \forall s^{\prime} \geq s\right\} \text {. }
$$

Since $\lim _{z \rightarrow-\infty}(\phi(z), \psi(z))=(0,0), \lim _{z \rightarrow \infty}(\phi(z), \psi(z))=$ $\left(1, b_{0}-b_{2}\right)$ and by using (49), there exists constant $A_{1}>$ 0 such that

$$
\begin{gathered}
(u(x, z, t), v(x, z, t)) \geq\left(1-\gamma, b_{0}-b_{2}-\gamma\right) \\
\forall z \geq A_{1},(x, z, t) \in \mathbb{R}^{n} \times \mathbb{R}, \\
(u(x, z, t), v(x, z, t)) \leq(\gamma, \gamma) \\
\forall z \leq-A_{1},(x, z, t) \in \mathbb{R}^{n} \times \mathbb{R} .
\end{gathered}
$$

Note that one can assume that $\gamma \in\left(0, \min \left\{1 / 2,\left(b_{0}-\right.\right.\right.$ $\left.\left.\left.b_{2}-\gamma\right) / 2\right\}\right)$. Let $A=\max \left\{A_{1},\left(\left(z^{*}-z_{*}\right) / 2\right)\right\}$, then $(u(x$, $z, t), v(x, z, t)) \geq\left(1-\gamma, b_{0}-b_{2}-\gamma\right)$ for $z \geq A$ and $(u(x$, $z, t), v(x, z, t)) \leq(\gamma, \gamma)$ for $z \leq-A$. It is easy to see that $s_{*} \leq 2 A$ and $s_{*}>-\infty$. Assume that $s_{*}>0$ and call $S=$ $\left\{(x, z, t) \in \mathbb{R}^{n} \times \mathbb{R},-A \leq z \leq A\right\}$. If $\inf _{S}\left(u^{s_{*}}-u\right)>0$ and $\inf _{S}\left(v^{s_{*}}-v\right)>0$, then there exists $\eta_{0} \in\left(0, s_{*}\right)$ such that $\left(u^{s_{*}-\eta_{0}}, v^{s_{*}-\eta_{0}}\right) \geq(u, v)$ in $S$ for all $\eta \in\left[0, \eta_{0}\right]$. Denote 
$E=\left\{(x, z, t) \in \mathbb{R}^{n} \times \mathbb{R}, z>A\right\}, w=u^{s_{*}-\eta}-u$ and $\omega=v^{s_{*}-\eta}-v$, we see that $(w, \omega) \geq(0,0)$ on $\partial E$ and satisfies

$$
\begin{aligned}
& w_{t}-\Delta w+c w_{z} \\
&=f\left(u^{s_{*}-\eta}\right)-f(u)+\left(k_{1}+k_{2} u\right) \omega-k_{2}\left(b_{0}-v^{s_{1 *}-\eta}\right) w \\
& \geq\left(k_{1}+k_{2} u\right) \omega-k_{2}\left(b_{0}-v^{s_{*}-\eta}\right) w-B w \\
& \omega_{t}-\Delta \omega+c \omega_{z}=-\left(k_{1}+k_{2} u\right) \omega+k_{2}\left(b_{0}-v^{s_{*}-\eta}\right) w
\end{aligned}
$$

for some constant $B$ (remember that $f \in C^{1}(\mathbb{R})$ and $u^{s_{*}-\eta}$, $u, v^{s_{*}-\eta}$ and $v$ are bounded). The parabolic maximum principle implies that $(w(x, z, t), \omega(x, z, t)) \geq(0,0)$ in $E$. Similarly, we can prove that $(w(x, z, t), \omega(x, z, t)) \geq(0,0)$ for $z \leq-A$. Therefore, $\left(u^{s_{*}-\eta}, v^{s_{*}-\eta}\right) \geq(u, v)$ in $\mathbb{R}^{n} \times \mathbb{R}$ for all $\eta \in\left[0, \eta_{0}\right]$. This contradicts the minimality of $s_{*}$. It follows then that

$$
\inf _{S}\left(u^{s_{*}}-u\right)=0 \quad \text { or } \quad \inf _{S}\left(v^{s_{*}}-v\right)=0 .
$$

We first consider the case that $\inf _{S}\left(u^{s_{*}}-u\right)=0$ and $\inf _{S}\left(v^{s_{*}}-\right.$ $v)>0$. As a consequence, there exist $z_{\infty} \in[-A, A]$ and a sequence $\left(x_{n}, z_{n}, t_{n}\right)_{n \in N}$ such that

$$
\begin{gathered}
z_{n} \longrightarrow z_{\infty}, \\
u^{s_{*}}\left(x_{n}, z_{n}, t_{n}\right)-u\left(x_{n}, z_{n}, t_{n}\right) \longrightarrow 0 \quad \text { as } n \longrightarrow \infty .
\end{gathered}
$$

Call $u_{n}(x, z, t)=u\left(x+x_{n}, z+z_{n}, t+t_{n}\right)$ and $v_{n}(x, z, t)=$ $v\left(x+x_{n}, z+z_{n}, t+t_{n}\right)$. Note that $v_{n}(x, z, t)$ is a bounded sequence. Up to extraction of a subsequence, the functions $\left(u_{n}, v_{n}\right)$ converge locally uniformly to a solution $\left(u_{\infty}\right.$, $\left.v_{\infty}\right)$ of the following system:

$$
\begin{gathered}
u_{t}=\Delta u-c u_{z}+f(u)+k_{1} v-k_{2} u\left(b_{0}-v\right), \\
v_{t}=\Delta v-c v_{z}-k_{1} v+k_{2} u\left(b_{0}-v\right) .
\end{gathered}
$$

It is easy to see that

$$
\begin{gathered}
w(x, z, t)=u_{\infty}\left(x+y, z+s_{*}, t+T\right)-u(x, z, t) \geq 0, \\
\omega(x, z, t)=v_{\infty}\left(x+y, z+s_{*}, t+T\right) \\
-v(x, z, t)>0 \text { in } \mathbb{R}^{n} \times \mathbb{R}
\end{gathered}
$$

and $w\left(0, z_{\infty}, 0\right)=0$; that is, $w(x, z, t)$ attains the local minimum at $\left(0, z_{\infty}, 0\right)$. We only consider the equation of $u$. It is easy to verify that $w$ cannot attain the local minimum because

$$
\begin{gathered}
w_{t}\left(0, z_{\infty}, 0\right)=0, \\
\Delta w\left(0, z_{\infty}, 0\right) \geq 0, \\
w_{z}\left(0, z_{\infty}, 0\right)=0, \\
\omega\left(0, z_{\infty}, 0\right)>0 .
\end{gathered}
$$

So we get a contradiction. Similarly, one can deal with the other case: $\inf _{S}\left(v^{s_{*}}-v\right)=0$ and $\inf _{S}\left(u^{s_{*}}-u\right)>0$.
Now we consider the last case: $\inf _{S}\left(u^{s_{*}}-u\right)=$ 0 and $\inf _{S}\left(v^{s_{*}}-v\right)=0$. As the case that $\inf _{S}\left(u^{s_{*}}-u\right)=$ 0 and $\inf _{S}\left(v^{s_{*}}-v\right)>0$, we obtain a solution $\left(u_{\infty}, v_{\infty}\right)$ of system (59) and $\left(w\left(0, z_{\infty}, 0\right), \omega\left(0, z_{\infty}, 0\right)\right)=0$. It follows from the strong parabolic maximum principle that $(w(x$, $z, t), \omega(x, z, t))=(0,0)$ for all $t \leq 0$ and then $(w(x$, $z, t), \omega(x, z, t)) \equiv(0,0)$ in $\mathbb{R}^{n} \times \mathbb{R}$ by uniqueness of the solution of the Cauchy problem (31). Thus, $u_{\infty}(0,0,0)=$ $u_{\infty}\left(k y, k s_{*}, k T\right)$ for all $k \in \mathbb{Z}$. But $u_{\infty}\left(k y, k s_{*}, k T\right) \rightarrow$ 1 as $k \rightarrow \infty$ since $s_{*}>0$. This is a contradiction.

Thus, $s_{*} \leq 0$,

$$
\begin{gathered}
u^{0}(x, z, t)=u(x+y, z, t+T) \geq u(x, z, t), \\
v^{0}(x, z, t)=v(x+y, z, t+T) \geq v(x, z, t) .
\end{gathered}
$$

Since $T \in \mathbb{R}$ and $y \in \mathbb{R}^{n-1}$ are arbitrary, we conclude that $u$ and $v$ depend on $z$ only, namely, $(u(x, z, t), v(x$, $z, t))=\left(\phi\left(z-z_{1}\right), \psi\left(z-z_{2}\right)\right)$ for some $z_{i} \in \mathbb{R}(i=1,2)$. Note that $(\phi(z), \psi(z))$ is a solution of (59), we conclude that $z_{1}=z_{2}=z_{0}$. This completes the proof.

From Lemma 9, any $\omega$-limit point of $(u, v)$ satisfies

$$
\begin{gathered}
\phi\left(z-z^{*}\right) \leq w(x, z, t) \leq \phi\left(z-z_{*}\right), \\
\psi\left(z-z^{*}\right) \leq \omega(x, z, t) \leq \psi\left(z-z_{*}\right), \quad(x, z) \in \mathbb{R}^{n}, t \in \mathbb{R},
\end{gathered}
$$

for some constant $z^{*}, z_{*} \in \mathbb{R}$. Combining the above lemma, we immediately have the following result.

Lemma 13. Let $(u(x, z, t), v(x, z, t))$ be a solution of $(31)$. Then any $\omega$-limit point $(w(x, z, t), \omega(x, z, t))$ of $(u, v)$ is a planar wave; that is, there exists a constant $z_{0} \mathbb{R}$ such that

$$
\begin{aligned}
& (w(x, z, t), \omega(x, z, t)) \\
& \quad=\left(\phi\left(z-z_{0}\right), \psi\left(z-z_{0}\right)\right), \quad(x, z) \in \mathbb{R}^{n}, t \in \mathbb{R} .
\end{aligned}
$$

Now, we derive estimate for the derivatives of the solution of (31).

Lemma 14 (monotonicity in $z$ ). Let $(u(x, z, t), v(x, z, t))$ be a solution of (31). Then for any constant $R>0$, there exists a constant $T>0$ such that

$$
\inf _{x \in \mathbb{R}^{n-1},|z| \leq R, t \geq T}\left(u_{z}(x, z, t), v_{z}(x, z, t)\right)>(0,0) .
$$

Proof. We prove this lemma by contradiction. If the above claim does not hold, then there exists a sequence $\left\{\left(x_{k}, z_{k}\right.\right.$, $\left.\left.t_{k}\right)\right\}$ such that $\left\{z_{k}\right\} \subset[-R, R], 0<t_{1}<t_{2}<\cdots \rightarrow \infty$ and that

$$
\liminf _{k \rightarrow \infty}\left(u_{z}\left(x_{k}, z_{k}, t_{k}\right), v_{z}\left(x_{k}, z_{k}, t_{k}\right)\right) \leq(0,0) .
$$

Replace $\left\{\left(x_{k}, z_{k}, t_{k}\right)\right\}$ by its subsequence if necessary; we may assume without loss of generality that $z_{k}$ converges to some limit $z_{\infty} \in[-R, R]$ and that

$$
\begin{array}{r}
\left(u_{z}, v_{z}\right)\left(x+x_{k}, z+z_{k}, t+t_{k}\right) \longrightarrow(w, \omega)(x, z, t) \\
\text { as } k \longrightarrow \infty \text { in } C_{\text {loc }}^{2,1}\left(\mathbb{R}^{n} \times \mathbb{R}\right),
\end{array}
$$


where $(w, \omega)$ is a limit point of $(u, v)$. Hence,

$$
\begin{aligned}
& \left(w_{z}\left(0, z_{\infty}, 0\right), \omega_{z}\left(0, z_{\infty}, 0\right)\right) \\
& \quad=\lim _{k \rightarrow \infty}\left(u_{z}\left(x_{k}, z_{k}, t_{k}\right), v_{z}\left(x_{k}, z_{k}, t_{k}\right)\right) \leq(0,0) .
\end{aligned}
$$

On the other hand, Lemma 13 shows that $(w(x, z, t), \omega(x$, $z, t))=\left(\phi\left(z-z_{0}\right), \psi\left(z-z_{0}\right)\right)$ for some $z_{0} \in \mathbb{R}$. This gets a contradiction because $\phi^{\prime}, \psi^{\prime}>0$. The proof of the lemma is completed.

By using Lemmas 14 and 9, the following corollary is obtained.

Corollary 15. Let $(u(x, z, t), v(x, z, t))$ be a solution of (31). Then there exists a constant $T>0$ such that

$$
\inf _{(x, z, t) \in D}\left(u_{z}(x, z, t), v_{z}(x, z, t)\right)>0
$$

where

$$
\begin{gathered}
D=\left\{(x, z, t) \in \mathbb{R}^{n} \times[T, \infty),\left(\frac{\phi(0)}{2}, \frac{\psi(0)}{2}\right)\right. \\
\leq(u(x, z, t), v(x, z, t)) \\
\left.\leq\left(\frac{1+\phi(0)}{2}, \frac{b_{0}-b_{2}+\psi(0)}{2}\right)\right\} .
\end{gathered}
$$

Lemma 16 (decay of $x$-derivatives). Let $(u(x, z, t), v(x, z$, $t)$ ) be a solution of (31). Then for any constant $R>0$, it holds that

$$
\begin{aligned}
& \lim _{t \rightarrow \infty} \sup _{x \in \mathbb{R}^{n-1},|z| \leq R}\left|u_{x_{i}}(x, z, t)\right|=0, \\
& \lim _{t \rightarrow \infty} \sup _{x \in \mathbb{R}^{n-1},|z| \leq R}\left|u_{x_{i} x_{j}}(x, z, t)\right|=0, \\
& \lim _{t \rightarrow \infty} \sup _{x \in \mathbb{R}^{n-1},|z| \leq R}\left|v_{x_{i}}(x, z, t)\right|=0, \\
& \lim _{t \rightarrow \infty} \sup _{x \in \mathbb{R}^{n-1},|z| \leq R}\left|v_{x_{i} x_{j}}(x, z, t)\right|=0,
\end{aligned}
$$

for each $1 \leq i, j \leq n-1$.

The proof of this lemma is similar to Lemma 4.9 in [11], and we omit it here.

Next we study the $(\phi(0), \psi(0))$-level surface of the solution of (31). From Corollary 15 and Lemma 16, we can derive the following lemma that the $(\phi(0), \psi(0))$-level surface of the solution $(u(x, z, t), v(x, z, t))$ has a graphical representation $z=\Gamma(x, t)$ for all $t$.

Lemma 17. Let $(u(x, z, t), v(x, z, t))$ be a solution of (31) and let $T>0$ be as defined in Corollary 15. Then there exists a smooth bounded function $\Gamma(x, t)$ such that

$$
\begin{gathered}
(u(x, z, t), v(x, z, t))=(\phi(0), \psi(0)), \\
\text { if and only if } z=\Gamma(x, t)
\end{gathered}
$$

for any $(x, t) \in \mathbb{R}^{n-1} \times[T, \infty)$. Furthermore, the following estimates hold: (i) for each $1 \leq i, j \leq n-1$,

$$
\begin{aligned}
& \lim _{t \rightarrow \infty} \sup _{x \in \mathbb{R}^{n-1}}\left|\Gamma_{x_{i}}(x, z, t)\right|=0, \\
& \lim _{t \rightarrow \infty} \sup _{x \in \mathbb{R}^{n-1}}\left|\Gamma_{x_{i} x_{j}}(x, z, t)\right|=0 ;
\end{aligned}
$$

(ii) there exists a constant $M>0$ such that, for each $1 \leq$ $i, j, k \leq n-1$,

$$
\sup _{x \in \mathbb{R}^{n-1}}\left|\Gamma_{x_{i} x_{j} x_{k}}(x, z, t)\right| \leq M \quad \text { for } t \geq T \text {. }
$$

Proof. Since

$$
\begin{gathered}
D=\left\{(x, z, t) \in \mathbb{R}^{n} \times[T, \infty),\left(\frac{\phi(0)}{2}, \frac{\psi(0)}{2}\right)\right. \\
\leq(u(x, z, t), v(x, z, t)) \\
\left.\leq\left(\frac{1+\phi(0)}{2}, \frac{b_{0}-b_{2}+\psi(0)}{2}\right)\right\}
\end{gathered}
$$

is bounded in the $z$-direction by virtue of Lemma 9 and the facts $(\phi, \psi)(-\infty)=(0,0)$ and $(\phi, \psi)(+\infty)=\left(1, b_{0}-\right.$ $\left.b_{2}\right)$, we can define a bounded function $\Gamma(x, t)$ satisfying $(72)$ thanks to Corollary 15 . Here $\Gamma(x, t)$ is smooth by the implicit function theorem, since $u(x, z, t)$ is smooth for $t>0$. The other estimates follow from Lemma 16, and we omit it here. This completes the proof.

The following lemma shows that the large time behavior of the solution can be essentially determined by the $(\phi(0)$, $\psi(0))$-level surface $\Gamma(x, t)$.

Lemma 18. Let $(u(x, z, t), v(x, z, t))$ be a solution of (31) and let $\Gamma(x, t)$ be as defined in Lemma 17. Then, it holds that

$$
\begin{aligned}
& \lim _{t \rightarrow \infty} \sup _{(x, z) \in \mathbb{R}^{n}}|u(x, z, t)-\phi(z-\Gamma(x, t))|=0, \\
& \lim _{t \rightarrow \infty} \sup _{(x, z) \in \mathbb{R}^{n}}|v(x, z, t)-\psi(z-\Gamma(x, t))|=0 .
\end{aligned}
$$

Proof. We prove this lemma by contradiction, and we only consider $u$. If the above claim does not hold, there exist a constant $\delta>0$ and a sequence $\left\{\left(x_{k}, z_{k}, t_{k}\right)\right\}$ such that $0<$ $t_{1}<t_{2}<\cdots \rightarrow \infty$ and that

$$
\left|u\left(x_{k}, z_{k}, t_{k}\right)-\phi\left(z_{k}-\Gamma\left(x_{k}, t_{k}\right)\right)\right| \geq \delta .
$$

On the other hand, by virtue of Lemma 9 and boundedness of $\Gamma(x, t)$, we can choose constants $R>0$ and $T>0$ such that

$$
\sup _{x \in \mathbb{R}^{n-1},|z| \geq R, t \geq T}|u(x, z, t)-\phi(z-\Gamma(x, t))|<\delta,
$$


which means that $\left\{z_{k}\right\}$ is bounded. We can choose subsequence of $\left\{\left(x_{k}, z_{k}, t_{k}\right)\right\}$, which we denote again by $\left\{\left(x_{k}, z_{k}\right.\right.$, $\left.\left.t_{k}\right)\right\}$ such that

$$
\begin{gathered}
z_{\infty}:=\lim _{k \rightarrow \infty} z_{k}, \\
\gamma_{\infty}:=\lim _{k \rightarrow \infty} \Gamma\left(x_{k}, t_{k}\right) \\
u\left(x+x_{k}, z, t+t_{k}\right) \longrightarrow w(x, z, t) \\
\text { as } k \longrightarrow \infty \text { in } C_{\text {loc }}^{2,1}\left(\mathbb{R}^{n} \times \mathbb{R}\right),
\end{gathered}
$$

where $w$ is some $\omega$-limit point of $u$. This and (77) show that

$$
\begin{aligned}
& \left|w\left(0, \gamma_{\infty}, 0\right)-\phi\left(z_{\infty}-\gamma_{\infty}\right)\right| \\
& \quad=\lim _{k \rightarrow \infty}\left|u\left(x_{k}, z_{k}, t_{k}\right)-\phi\left(z_{k}-\gamma\left(x_{k}, t_{k}\right)\right)\right| \geq \delta .
\end{aligned}
$$

On the other hand, we have

$$
w\left(0, \gamma_{\infty}, 0\right)=\lim _{k \rightarrow \infty} u\left(x_{k}, \Gamma\left(x_{k}, t_{k}\right), t_{k}\right)=\phi(0) .
$$

Lemma 13 implies that $w(x, z, t) \equiv \phi\left(z-\gamma_{\infty}\right)$. This contradicts (80). This completes the proof of this lemma.

By setting $y=z-c t$ and $\gamma(x, t)=\Gamma(x, t)-c t$, we obtain the statement (i), (ii) of Theorem 1 from Lemmas 17 and 18. Thus, it remains to prove the statement (iii). This will be done at the end of this section.

In the following, we construct supersolutions of (31). For this purpose, we consider the problem of the form

$$
\begin{gathered}
V_{t}=\Delta_{x} V-\frac{c}{2}\left|\nabla_{x} V\right|^{2}, \quad x \in \mathbb{R}^{n-1}, t>0, \\
v(x, 0)=v_{0}(x), \quad x \in \mathbb{R}^{n-1} .
\end{gathered}
$$

Lemma 19 (supersolution). For any constant $M>0$, there exist positive constant $\delta, \varepsilon_{0}, k_{0}$ and smooth functions $p(t)$ and $q(t)$ satisfying

$$
\begin{aligned}
p(0) & >0, \\
q(0) & =0, \\
0 \leq p(t), \quad q(t) & \leq \varepsilon \quad \text { for } t \geq 0,
\end{aligned}
$$

where $\varepsilon \in\left(0, \varepsilon_{0}\right]$, such that if $V(x, t)$ is any solution of (82) with $\left\|V_{0}\right\|_{W^{3, \infty}} \leq M$ and $\left\|\nabla_{x} V_{0}\right\|_{W^{1, \infty}} \leq \delta$, then the function defined by

$$
\begin{gathered}
u^{+}(x, z, t)=\phi\left(\frac{z-V(x, t)}{\sqrt{1+\left|\nabla_{x} V\right|^{2}}}+q(t)\right)+p(t), \\
v^{+}(x, z, t)=\psi\left(\frac{z-V(x, t)}{\sqrt{1+\left|\nabla_{x} V\right|^{2}}}+q(t)\right)+k_{0} p(t)
\end{gathered}
$$

satisfies

$$
\begin{aligned}
L\left[u^{+}\right]:= & u_{t}^{+}-\Delta u^{+}+c u_{z}^{+}-f\left(u^{+}\right)-k_{1} v^{+} \\
& +k_{2} u^{+}\left(b_{0}-v^{+}\right) \geq 0, \quad(x, z) \in \mathbb{R}^{n}, t>0, \\
L\left[v^{+}\right]:= & v_{t}^{+}-\Delta v^{+}+c v_{z}^{+}+k_{1} v^{+} \\
& -k_{2} u^{+}\left(b_{0}-v^{+}\right) \geq 0, \quad(x, z) \in \mathbb{R}^{n}, t>0 .
\end{aligned}
$$

Proof. We divide the proof into four steps. The first two steps are similar to that of Lemma 4.12 in [11], and we only give the last results.

Step 1. Set

$$
\eta(x, z, t)=\frac{z-V(x, t)}{\sqrt{1+\left|\nabla_{x} V\right|^{2}}} .
$$

Then, by using (8), we have

$$
\begin{aligned}
L\left[u^{+}\right]= & \left(\eta_{t}-\Delta_{x} \eta+c \eta_{z}-c\right) \phi^{\prime}+\left(1-\left|\nabla_{x} \eta\right|^{2}-\eta_{z}^{2}\right) \phi^{\prime \prime} \\
& +q^{\prime}(t) \phi^{\prime}+p^{\prime}(t)-f(\phi+p(t)) \\
& +f(\phi)-\left(k_{1}+k_{2} \phi\right) k_{0} p(t) \\
& +k_{2} p(t)\left(b_{0}-\psi-k_{0} p(t)\right) \\
L\left[v^{+}\right]= & \left(\eta_{t}-\Delta_{x} \eta+c \eta_{z}-c\right) \psi^{\prime} \\
& +\left(1-\left|\nabla_{x} \eta\right|^{2}-\eta_{z}^{2}\right) \psi^{\prime \prime}+q^{\prime}(t) \psi^{\prime} \\
& +k_{0} p^{\prime}(t)+\left(k_{1}+k_{2} \phi\right) k_{0} p(t) \\
& -k_{2} p(t)\left(b_{0}-\psi-k_{0} p(t)\right) .
\end{aligned}
$$

By rewriting the above expression in terms of $V$, we obtain

$$
\begin{aligned}
L\left[u^{+}\right]= & \left(I_{0}-I_{2}\right) \phi^{\prime}+\left(I_{1}-3 I_{3}\right) \eta \phi^{\prime}-2 I_{2} \eta \phi^{\prime \prime} \\
& -I_{3} \eta^{2} \phi^{\prime \prime}+J_{1}, \\
L\left[v^{+}\right]= & \left(I_{0}-I_{2}\right) \psi^{\prime}+\left(I_{1}-3 I_{3}\right) \eta \psi^{\prime}-2 I_{2} \eta \psi^{\prime \prime} \\
& -I_{3} \eta^{2} \psi^{\prime \prime}+J_{2},
\end{aligned}
$$

where $I_{i}, J_{j}(i=0,1,2,3 ; j=1,2)$ are functions given by

$$
\begin{gathered}
I_{0}=-\frac{V_{t}-c}{\sqrt{1+\left|\nabla_{x} V\right|^{2}}}+\operatorname{div}\left(\frac{\nabla_{x}}{\sqrt{1+\left|\nabla_{x} V\right|^{2}}}\right)-c, \\
I_{1}=-\sum_{i=1}^{n-1} \frac{V_{x_{i}} V_{x_{i t}}}{1+\left|\nabla_{x} V\right|^{2}}+\sum_{i, j=1}^{n-1} \frac{V_{x_{i} x_{j}}^{2}+V_{x_{j}} V_{x_{i} x_{i} x_{j}}}{1+\left|\nabla_{x} V\right|^{2}},
\end{gathered}
$$




$$
\begin{gathered}
I_{2}=\sum_{i, j=1}^{n-1} \frac{V_{x_{i}} V_{x_{j}}+V_{x_{j}} V_{x_{i} x_{j}}}{\left(1+\left|\nabla_{x} V\right|^{2}\right)^{3 / 2}} \\
I_{3}=\sum_{i=1}^{n-1}\left(\frac{\sum_{i=1}^{n-1} V_{x_{i}} V_{x_{i j}}}{1+\left|\nabla_{x} V\right|^{2}}\right), \\
J_{1}=\left(\phi^{\prime} \frac{q^{\prime}(t)}{p(t)}+\frac{p^{\prime}(t)}{p(t)}\right. \\
-\int_{0}^{1} f^{\prime}(\phi+\tau p(t)) d \tau-\left(k_{1}+k_{2} \phi\right) k_{0} \\
\left.+k_{2}\left(b_{0}-\psi-k_{0} p(t)\right)\right) \cdot p(t), \\
J_{2}=\left(\psi^{\prime} \frac{q^{\prime}(t)}{k_{0} p(t)}+\frac{p^{\prime}(t)}{p(t)}+\left(k_{1}+k_{2} \phi\right)\right. \\
\left.+k_{2}\left(b_{0}-\psi-k_{0} p(t)\right) \frac{1}{k_{0}}\right) \cdot k_{0} p(t) .
\end{gathered}
$$

Step 2. Now we estimate $I_{i}(i=1,2,3)$. Define $I_{u}(x, z, t)$ and $I_{v}(x, z, t)$ by

$$
\begin{aligned}
& I_{u}(x, z, t)=\left(I_{0}-I_{2}\right) \phi^{\prime}+\left(I_{1}-3 I_{3}\right) \eta \phi^{\prime}-2 I_{2} \eta \phi^{\prime \prime}-I_{3} \eta^{2} \phi^{\prime \prime}, \\
& I_{v}(x, z, t)=\left(I_{0}-I_{2}\right) \psi^{\prime}+\left(I_{1}-3 I_{3}\right) \eta \psi^{\prime}-2 I_{2} \eta \psi^{\prime \prime}-I_{3} \eta^{2} \psi^{\prime \prime} .
\end{aligned}
$$

It follows from Theorem 2.1 in [5] that $\phi^{\prime}(z), \phi^{\prime \prime}(z), \psi^{\prime \prime}(z)$ and $\psi^{\prime \prime}(z)$ decay to zero exponentially as $|z| \rightarrow \infty$. Noting that $q(t)$ is assumed to be bounded, the following functions are all bounded:

$$
\begin{gathered}
\eta \phi^{\prime}(\eta+q(t)), \\
\eta \phi^{\prime \prime}(\eta+q(t)), \\
\eta^{2} \phi^{\prime \prime}(\eta+q(t)), \\
\eta \psi^{\prime}(\eta+q(t)), \\
\eta \psi^{\prime \prime}(\eta+q(t)), \\
\eta^{2} \psi^{\prime \prime}(\eta+q(t)) .
\end{gathered}
$$

Now we choose a constant $C_{1}>0$ arbitrarily. Then from the above boundedness and Lemma 8 , we can choose a constant $C_{2} \geq 1$ depending only on $c$ and $M$, and a constant $\delta>0$ depending only on $C_{1}$ such that if $\left\|\nabla_{x} V_{0}\right\|_{W^{1, \infty}} \leq$ $\delta$, it holds that

$$
\begin{gathered}
\left|I_{u}(x, z, t)\right| \leq P(t), \\
\left|I_{v}(x, z, t)\right| \leq P(t) \quad \text { where } P(t)=\min \left\{C_{2} t^{-2}, C_{1}\right\} .
\end{gathered}
$$

Step 3. Now we determine the constant $C_{1}, \varepsilon_{0}$ and the smooth functions $p_{i}(t)$ and $q(t)(i=1,2)$. Set

$$
\begin{gathered}
F(u, v)=f(u)+k_{1} v-k_{2} u\left(b_{0}-v\right), \\
G(u, v)=-k_{1} v+k_{2} u\left(b_{0}-v\right) .
\end{gathered}
$$

Then, direct calculation shows that

$$
\begin{gathered}
F_{u}=f^{\prime}(u)-k_{2}\left(b_{0}-v\right), \\
F_{v}=k_{1}+k_{2} u, \\
G_{u}=k_{2}\left(b_{0}-v\right), \\
G_{v}=-\left(k_{1}+k_{2} u\right), \\
F_{u}(0,0) G_{v}(0,0)-F_{v}(0,0) G_{u}(0,0)=-k_{1} f^{\prime}(0)>0, \\
F_{u}\left(1, b_{0}-b_{2}\right) G_{v}\left(1, b_{0}-b_{2}\right)-F_{v}\left(1, b_{0}-b_{2}\right) G_{u}\left(1, b_{0}-b_{2}\right) \\
=-\left(k_{1}+k_{2}\right) f^{\prime}(1)>0 .
\end{gathered}
$$

Hence, there exist constants $\varepsilon_{0}>0, \alpha_{11}, \alpha_{22}<0$ and $\alpha_{12}$, $\alpha_{21}>0$ such that

$$
\begin{aligned}
& \alpha_{11}<F_{u}<0, \\
& \alpha_{22}<G_{v}<0, \\
& 0<F_{v}<\alpha_{12}, \\
& 0<G_{v}<\alpha_{21}, \\
& \text { on } A_{\varepsilon}^{i}, i=1,2,
\end{aligned}
$$

where $\varepsilon \in\left(0, \varepsilon_{0}\right]$ and

$$
\begin{gathered}
A_{\varepsilon}^{0}=\{(u, v)|| u|\leq \varepsilon,| v \mid \leq \varepsilon\}, \\
A_{\varepsilon}^{1}=\left\{(u, v)|| u-1|\leq \varepsilon,| v-b_{0}+b_{2} \mid \leq \varepsilon\right\} .
\end{gathered}
$$

It follows that there exist positive constants $K_{0}$ and $k_{0}$ such that for all $K \in\left(0, K_{0}\right)$, we have

$$
\begin{gathered}
2 K+\alpha_{11}<0, \\
2 K+\alpha_{22}<0, \\
\left(2 K+\alpha_{11}\right)\left(2 K+\alpha_{22}\right)>\alpha_{12} \alpha_{21}, \\
-\frac{\alpha_{21}}{2 K+\alpha_{22}}<k_{0}<-\frac{2 K+\alpha_{11}}{\alpha_{12}} .
\end{gathered}
$$

This implies that

$$
\begin{aligned}
& -2 K-\alpha_{11}-k_{0} \alpha_{12}>0 \\
& -2 K-\alpha_{22}-\frac{1}{k_{0}} \alpha_{21}>0 .
\end{aligned}
$$

Denote $\xi=(z-V(x, t)) / \sqrt{1+\left|\nabla_{x} V\right|^{2}}+q(t)$. Using the above estimates and the facts that $\lim _{z \rightarrow-\infty} \phi(z)=0$, 
$\lim _{z \rightarrow-\infty} \psi(z)=0, \lim _{z \rightarrow \infty} \phi(z)=1$ and $\lim _{z \rightarrow \infty} \psi(z)=$ $b_{0}-b_{2}$, there exist constants $M_{1}, M_{2}>0$ such that

$$
\begin{gathered}
-2 K-f^{\prime}\left(\phi+\theta \delta_{1} e^{-\beta t}\right)-k_{0}\left(k_{1}+k_{2} \phi\right)+k_{2}\left(b_{0}-\psi\right)>0 \\
\qquad \text { for } \xi \leq-M_{2} \text { or } \xi \geq M_{1}, \\
-2 K+k_{1}+k_{2} \phi-k_{2} \frac{1}{k_{0}}\left(b_{0}-\psi\right)>0 \\
\text { for } \xi \leq-M_{2} \text { or } \xi \geq M_{1} .
\end{gathered}
$$

Set

$$
\delta_{0}=\min \left\{\frac{-2 K-\alpha_{11}-k_{0} \alpha_{12}}{2 k_{2} k_{0}}, \frac{-2 K-\alpha_{22}-\alpha_{21} / k_{0}}{2 k_{2} k_{0}}, 1, \frac{1}{k_{0}}\right\} .
$$

We define the constant $C_{1}>0$ and $C_{0} \geq 1$ by

$$
\begin{aligned}
& C_{1}=\frac{K^{2} \varepsilon^{2} k_{0}^{2}}{16\left(1+k_{0}\right)^{2} C_{2} C_{0}^{2}}, \\
& C_{0}=\max \left\{1, \frac{2 K+\left\|f^{\prime}\right\|_{L^{\infty}([0,1])}+\left(k_{1}+k_{2}\right) k_{0}+\delta_{0} k_{2}}{\inf _{-M_{2} \leq \xi \leq M_{1}} \phi^{\prime}(\xi)},\right. \\
& \left.\frac{2 K k_{0}-k_{1} k_{0}+k_{2} b_{0}}{\inf _{-M_{2} \leq \xi \leq M_{1}} \psi^{\prime}(\xi)}\right\}
\end{aligned}
$$

We choose functions $p(t), q(t) \in C^{\infty}[0, \infty)$ satisfying

$$
\begin{gathered}
P(t) \leq \min \left\{1, k_{0}\right\} K p(t), \\
K p(t) \leq 2\left(1+\frac{1}{k_{0}}\right) P(t), \\
K\left|p^{\prime}(t)\right| \leq 2\left|P^{\prime}(t)\right|, \\
q(t)=\int_{0}^{t} p(s) d s .
\end{gathered}
$$

Then (107) holds, since we have

$$
\begin{gathered}
p(0) \geq \frac{K \varepsilon^{2} k_{0}}{16\left(1+k_{0}\right)^{2} C_{2} C_{0}^{2}}>0, \\
0<p(t) \leq \frac{K \varepsilon^{2} k_{0}}{8 C_{2} C_{0}^{2}\left(1+k_{0}\right)} \leq \varepsilon \\
0 \leq \int_{0}^{\infty} p(s) d s \leq \varepsilon .
\end{gathered}
$$

Step 4. Now we complete the proof. Since $\left|I_{u}(x, z, t)\right| \leq$ $P(t)$ and $\left|I_{v}(x, z, t)\right| \leq P(t)$ by Step 2 , it suffices to show the inequality $J_{i}(x, z, t) \geq P(t)(i=1,2)$. Letting $\xi=(z-$
$V(x, t)) / \sqrt{1+\left|\nabla_{x} V\right|^{2}}+q(t)$, when $\xi \in\left(-\infty,-M_{2}\right] \cup\left[M_{1}, \infty\right)$, we have

$$
\begin{gathered}
J_{1} \geq\left(\frac{p^{\prime}(t)}{p(t)}-\int_{0}^{1} f^{\prime}(\phi+\tau p(t)) d \tau\right. \\
\left.\quad-\left(k_{1}+k_{2}\right) k_{0}+k_{2}\left(b_{0}-\psi-p(t)\right)\right) \cdot p(t) \\
\geq K p(t) \geq P(t), \\
J_{2} \geq\left(\frac{p^{\prime}(t)}{p(t)}+\left(k_{1}+k_{2} \phi\right)+k_{2}\left(b_{0}-\psi-p(t)\right) \frac{1}{k_{0}}\right) \cdot k_{0} p(t) \\
\geq k_{0} K p(t) \geq P(t),
\end{gathered}
$$

since we have

$$
\sup _{t \geq 0} \frac{\left|p^{\prime}(t)\right|}{p(t)} \leq \sup _{t \geq 0} \frac{2\left|P^{\prime}(t)\right|}{K p(t)} \leq \sup _{t \geq 0} \frac{2\left|P^{\prime}(t)\right|}{P(t)}=\frac{K \varepsilon}{C_{0}}<K .
$$

For any $-M_{2} \leq \xi \leq M_{1}$, we have

$$
\begin{gathered}
L\left[u^{+}\right] \geq\left(C_{0} \inf _{-M_{2} \leq \xi \leq M_{1}} \phi^{\prime}(\xi)-2 K-\left\|f^{\prime}\right\|_{L^{\infty}([0,1])}\right. \\
\left.-\left(k_{1}+k_{2}\right) k_{0}-\delta_{0} k_{2}\right) p(t) \geq 0,
\end{gathered}
$$

$$
L\left[v^{+}\right] \geq\left(\frac{C_{0}}{k_{0}} \inf _{-M_{2} \leq \xi \leq M_{1}} \psi^{\prime}(\xi)-2 K+k_{1}-\frac{k_{2} b_{0}}{k_{0}}\right) \geq 0 .
$$

In summary, we have $L\left[u^{+}\right] \geq 0$ and $L\left[u^{+}\right] \geq 0$. This completes the proof.

Lemma 20 (subsolution). For any constants $M>0$, there exist positive constant $\delta, \varepsilon_{0}, k_{0}$ and smooth functions $p(t)$ and $q(t)$ satisfying

$$
\begin{gathered}
p(0)>0, \\
q(0)=0, \\
0 \leq p(t), \quad q(t) \leq \varepsilon \quad \text { for } t \geq 0,
\end{gathered}
$$

where $\varepsilon \in\left(0, \varepsilon_{0}\right]$, such that if $V(x, t)$ is any solution of $(82)$ with $\left\|V_{0}\right\|_{W^{3, \infty}} \leq M$ and $\left\|\nabla_{x} V_{0}\right\|_{W^{1, \infty}} \leq \delta$, then the function defined by

$$
\begin{aligned}
& u^{-}(x, z, t)=\phi\left(\frac{z-V(x, t)}{\sqrt{1+\left|\nabla_{x} V\right|^{2}}}-q(t)\right)-p(t), \\
& v^{-}(x, z, t)=\psi\left(\frac{z-V(x, t)}{\sqrt{1+\left|\nabla_{x} V\right|^{2}}}-q(t)\right)-k_{0} p(t)
\end{aligned}
$$


satisfies

$$
\begin{aligned}
L\left[u^{-}\right]:= & u_{t}^{-}-\Delta u^{-}+c u_{z}^{-}-f\left(u^{-}\right) \\
& -k_{1} v^{-}+k_{2} u^{-}\left(b_{0}-v^{-}\right) \leq 0, \quad(x, z) \in \mathbb{R}^{n}, t>0, \\
L\left[v^{-}\right]:= & v_{t}^{-}-\Delta v^{-}+c v_{z}^{-}+k_{1} v^{-} \\
& -k_{2} u^{-}\left(b_{0}-v^{-}\right) \leq 0, \quad(x, z) \in \mathbb{R}^{n}, t>0 .
\end{aligned}
$$

The proof of Lemma 20 is similar to that of Lemma 19, and we omit it here.

Lemma 21 (approximation of $\Gamma(x, t))$. Let $(u(x, z, t), v(x$, $z, t)$ ) be a solution of (31) and let $\Gamma(x, t)$ be as defined in Lemma 17. Then for any $\varepsilon>0$, there exists a constant $\tau_{\varepsilon}>$ 0 such that the function $V(x, t)$ defined by

$$
\begin{gathered}
V_{t}=\Delta_{x} V-\frac{c}{2}\left|\nabla_{x} v_{1}\right|^{2}, \quad x \in \mathbb{R}^{n-1}, t>0, \\
v_{1}(x, 0)=\Gamma\left(x, \tau_{\varepsilon}\right), \quad x \in \mathbb{R}^{n-1}
\end{gathered}
$$

satisfies

$$
\sup _{x \in \mathbb{R}^{n-1}}\left|\Gamma(x, t) \geq V\left(x, t-\tau_{\varepsilon}\right)\right| \leq \varepsilon, \quad t \geq \tau_{\varepsilon} .
$$

Proof. From Corollary 15, we can choose constants $T>$ $0, M>0$, and $L>0$ such that

$$
\begin{gathered}
\sup _{t \geq T}\|\Gamma(\cdot, t)\|_{W^{3, \infty}} \leq M, \\
\inf _{(x, z, t) \in D} u_{z}(x, z, t) \geq L, \\
\inf _{(x, z, t) \in D} v_{z}(x, z, t) \geq L, \\
D=\left\{(x, z, t) \in \mathbb{R}^{n} \times[T, \infty),\left(\frac{\phi(0)}{2}, \frac{\psi(0)}{2}\right)\right. \\
\leq(u(x, z, t), v(x, z, t)) \\
\left.\leq\left(\frac{1+\phi(0)}{2}, \frac{b_{0}-b_{2}+\psi(0)}{2}\right)\right\} .
\end{gathered}
$$

For the constant $M$ and $\widehat{\varepsilon}:=\min \left\{\varepsilon_{0}, \min \{L \varepsilon,(1+\phi(0)) /\right.$ $\left.\left.\left[2\left(1+\left\|\phi^{\prime}\right\|_{L^{\infty}}\right)\right]\right\}\right\}$, we choose a constant $\delta>0$ and functions $p(t), q(t)$ satisfying

$$
\begin{aligned}
p(0) & >0, \\
q(0) & =0, \\
0 \leq p(t), \quad q(t) & \leq \widehat{\varepsilon} \quad \text { for } t \geq 0,
\end{aligned}
$$

as in Lemma 19. Then it follows from Lemma 17 that there exists a constant $\tau_{\varepsilon} \in[T, \infty)$ such that $\left\|\Gamma\left(\cdot, \tau_{\varepsilon}\right)\right\|_{W^{1, \infty}} \leq \delta$.
Moreover, using Lemma 18 , by choosing $\tau_{\varepsilon}$ larger if necessary, we have

$$
\begin{aligned}
u\left(x, z, \tau_{\varepsilon}\right) & \leq \phi\left(z-\Gamma\left(x, \tau_{\varepsilon}\right)\right)+\frac{p(0)}{2} \\
& \leq \phi\left(\frac{z-\Gamma\left(x, \tau_{\varepsilon}\right)}{\sqrt{1+\left|\nabla_{x} \Gamma\left(x, \tau_{\varepsilon}\right)\right|^{2}}}\right)+p(0), \\
v\left(x, z, \tau_{\varepsilon}\right) & \leq \psi\left(z-\Gamma\left(x, \tau_{\varepsilon}\right)\right)+\frac{k_{0} p(0)}{2} \\
& \leq \psi\left(\frac{z-\Gamma\left(x, \tau_{\varepsilon}\right)}{\sqrt{1+\left|\nabla_{x} \Gamma\left(x, \tau_{\varepsilon}\right)\right|^{2}}}\right)+k_{0} p(0),
\end{aligned}
$$

where we used the smallness of $\left|\nabla_{x} \Gamma\right|$. For such $\tau_{\varepsilon}$, we define $V(x, t)$ as a function satisfying (110). Then Lemma 19 shows that the function defined by

$$
\begin{aligned}
& u^{+}(x, z, t) \\
& \quad=\phi\left(\frac{z-V\left(x, t-\tau_{\varepsilon}\right)}{\sqrt{1+\left|\nabla_{x} V\right|^{2}}}+q\left(t-\tau_{\varepsilon}\right)\right)+p\left(t-\tau_{\varepsilon}\right), \\
& v^{+}(x, z, t) \\
& \quad=\psi\left(\frac{z-V\left(x, t-\tau_{\varepsilon}\right)}{\sqrt{1+\left|\nabla_{x} V\right|^{2}}}+q\left(t-\tau_{\varepsilon}\right)\right)+k_{0} p\left(t-\tau_{\varepsilon}\right)
\end{aligned}
$$

is a supersolution of (31) for $t \geq \tau_{\varepsilon}$. Since (114) implies that $u\left(x, z, \tau_{\varepsilon}\right) \leq u^{+}\left(x, z, \tau_{\varepsilon}\right)$ and $v\left(x, z, \tau_{\varepsilon}\right) \leq v^{+}\left(x, z, \tau_{\varepsilon}\right)$, the comparison principle gives that $u(x, z, t) \leq u^{+}(x, z, t)$ and $v(x, z, t) \leq v^{+}(x, z, t)$ for $t \geq \tau_{\varepsilon}$. Then, due to $p(t), q(t) \leq$ $\widehat{\mathcal{\varepsilon}}$, we have

$$
\begin{aligned}
u(x, & \left.V\left(x, t-\tau_{\varepsilon}\right), t\right)-\phi(0) \\
& \leq u^{+}\left(x, V\left(x, t-\tau_{\varepsilon}\right), t\right)-\phi(0) \\
& =\phi\left(q\left(t-\tau_{\varepsilon}\right)\right)+p(t)-\phi(0) \\
& \leq\left(1+\left\|\phi^{\prime}\right\|_{L^{\infty}}\right) \widehat{\varepsilon} \\
& \leq \min \left\{L \varepsilon, \frac{(1+\phi(0))}{2}\right\} .
\end{aligned}
$$

Noting that $u(x, \Gamma(x, t), t)=\phi(0)$, we get

$$
\begin{aligned}
L \varepsilon & \geq u\left(x, V\left(x, t-\tau_{\varepsilon}\right), t\right)-u\left(x, \Gamma\left(x, t-\tau_{\varepsilon}\right), t\right) \\
& \geq \inf _{u \in[\phi(0),(1+\phi(0)) / 2], t \geq \tau_{\varepsilon}} u_{z} \times(V(x, t)-\Gamma(x, t)) \\
& \geq L(V(x, t)-\Gamma(x, t)),
\end{aligned}
$$

which implies that $V(x, t-T)-\Gamma(x, t) \leq \varepsilon$ for $t>\tau_{\varepsilon}$. Similarly, by using the subsolution $\left(u^{-}, v^{-}\right)$given in Lemma 20 , 
we can show that $V(x, t-T)-\Gamma(x, t) \geq-\varepsilon$ for $t>\tau_{\varepsilon}$. This completes the proof.

Now we are ready to complete the proof of Theorem 1 .

Proof of Theorem 1. The statements (i) and (ii) of Theorem 1 are directly from Lemmas 17 and 18, respectively. Thus, we only prove the statement (iii).

By Lemma 21, the large time behavior of the $(\phi(0), \psi(0))$ level surface $\Gamma(x, t)$ of the solution $u(x, z, t)$ of (31) can be approximated by function $V(x, t)$ of the equation

$$
V_{t}=\Delta_{x} V-\frac{c}{2}\left|\nabla_{x} V\right|^{2}, \quad x \in \mathbb{R}^{n-1}, t>0 .
$$

This means that the $(\phi(0), \psi(0))$-level surface $\gamma(x, t)=$ $\Gamma(x, t)-c t$ of the solution $(u(x, y, t), v(x, y, t))$ of $(7)$ can be approximated by function $\widetilde{V}(x, t)$ of the equation

$$
\widetilde{V}_{t}=\Delta_{x} \widetilde{V}-\frac{c}{2}\left|\nabla_{x} \widetilde{V}\right|^{2}-c, \quad x \in \mathbb{R}^{n-1}, t>0 .
$$

Hence, the statement (iii) of Theorem 1 follows from Lemma 21. This completes the proof of Theorem 1.

Next, we prove Theorem 3. Firstly, similar to Lemma 4.15 in [11], one can prove that the $(\phi(0), \psi(0))$-level surface $\Gamma(x, t)$ remains uniquely ergodic for all large $t \geq 0$.

Lemma 22 (ergodicity of $(\phi(0), \psi(0))$-level surface). Let $(u(x, z, t), v(x, z, t))$ be a solution of (31) and assume that $\left(u_{0}(x, z), v_{0}(x, z)\right)$ is uniquely ergodic in the $x$-direction. Then the $(\phi(0), \psi(0))$-level surface $\Gamma(x, t)$ defined in Lemma 17 is uniquely ergodic for each $t \geq T$, where $T>0$ is the constant in Lemma 17.

Proof of Theorem 3. Let $T>0$ and $\Gamma(x, t)$ be as in Lemma 17 , then $\Gamma(x, t)$ is uniquely ergodic for each $t \geq T$ from Lemma 22. Combining Lemmas 6 and 7, one can easily prove Theorem 3.

\section{Acknowledgments}

The first author was supported in part by PRC Grants NSFC 11226168 and 11301146, and the second author was supported in part by PRC Grants NSFC 11171064, 11301146, and 11226168 and the Natural Science Foundation of Jiangsu province BK2011583. The authors are grateful to the referees for their valuable suggestions and comments on the original paper.

\section{References}

[1] J.-C. Tsai and J. Sneyd, "Existence and stability of traveling waves in buffered systems," SIAM Journal on Applied Mathematics, vol. 66, no. 1, pp. 237-265, 2005.

[2] J.-C. Tsai and J. Sneyd, "Are buffers boring? Uniqueness and asymptotical stability of traveling wave fronts in the buffered bistable system," Journal of Mathematical Biology, vol. 54, no. 4, pp. 513-553, 2007.

[3] J.-S. Guo and J.-C. Tsai, "The asymptotic behavior of solutions of the buffered bistable system," Journal of Mathematical Biology, vol. 53, no. 1, pp. 179-213, 2006.
[4] J. Sneyd, P. D. Dale, and A. Duffy, "Traveling waves in buffered systems: applications to calcium waves," SIAM Journal on Applied Mathematics, vol. 58, no. 4, pp. 1178-1192, 1998.

[5] G. Lv, "Stability of planar waves in the buffered bistable system," Mathematical Methods in the Applied Sciences, vol. 35, no. 9, pp. 1078-1088, 2012.

[6] H. Matano, M. Nara, and M. Taniguchi, "Stability of planar waves in the Allen-Cahn equation," Communications in Partial Differential Equations, vol. 34, no. 7-9, pp. 976-1002, 2009.

[7] G. Lv and M. Wang, "Stability of planar waves in monostable reaction-diffusion equations," Proceedings of the American Mathematical Society, vol. 139, no. 10, pp. 3611-3621, 2011.

[8] T. Kapitula, "Multidimensional stability of planar travelling waves," Transactions of the American Mathematical Society, vol. 349, no. 1, pp. 257-269, 1997.

[9] C. D. Levermore and J. X. Xin, "Multidimensional stability of traveling waves in a bistable reaction-diffusion equation. II," Communications in Partial Differential Equations, vol. 17, no. 1112, pp. 1901-1924, 1992.

[10] J. X. Xin, "Multidimensional stability of traveling waves in a bistable reaction-diffusion equation. I," Communications in Partial Differential Equations, vol. 17, no. 11-12, pp. 1889-1899, 1992.

[11] H. Matano and M. Nara, "Large time behavior of disturbed planar fronts in the Allen-Cahn equation," Journal of Differential Equations, vol. 251, no. 12, pp. 3522-3557, 2011.

[12] G. Lv, "Large time behavior of planar waves in monostablereaction-diffusion equations," submitted.

[13] M. Bramanti and L. Brandolini, "Schauder estimates for parabolic nondivergence operators of Hörmander type," Journal of Differential Equations, vol. 234, no. 1, pp. 177-245, 2007.

[14] W. Schlag, "Schauder and $L^{p}$ estimates for parabolic systems via Campanato spaces," Communications in Partial Differential Equations, vol. 21, no. 7-8, pp. 1141-1175, 1996. 


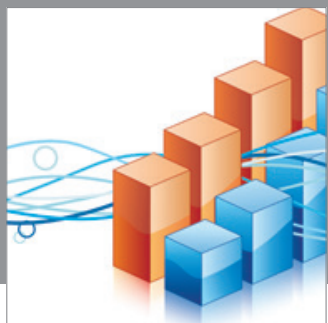

Advances in

Operations Research

mansans

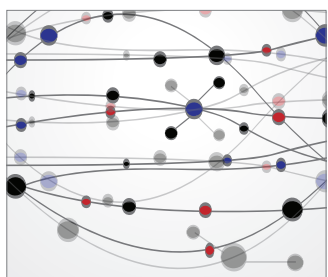

The Scientific World Journal
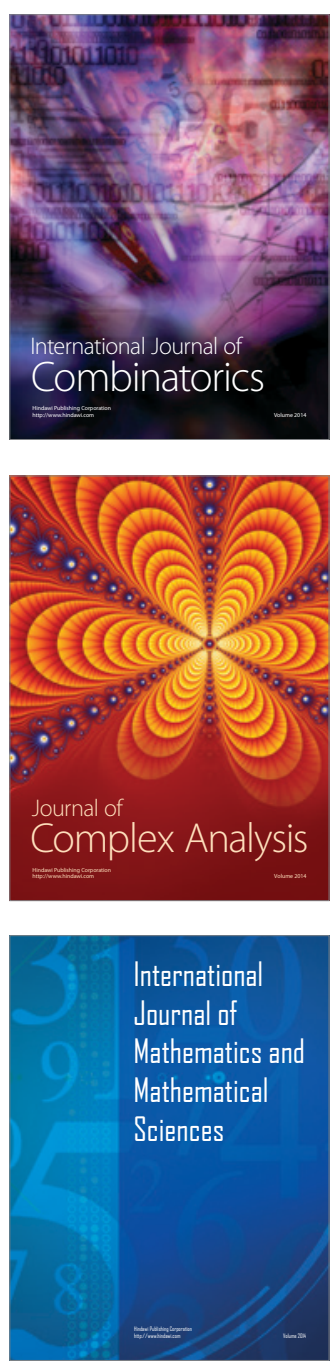
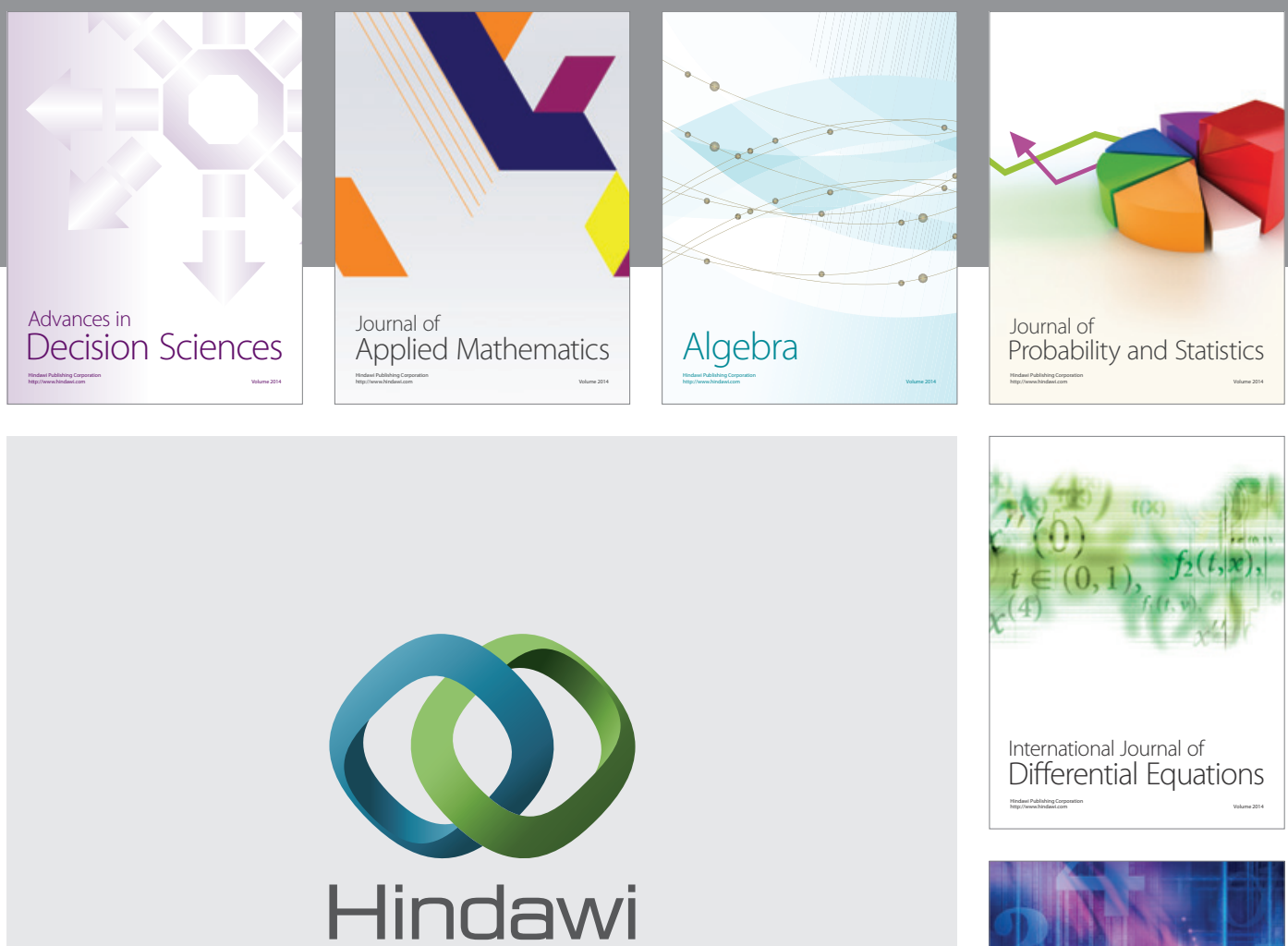

Submit your manuscripts at http://www.hindawi.com
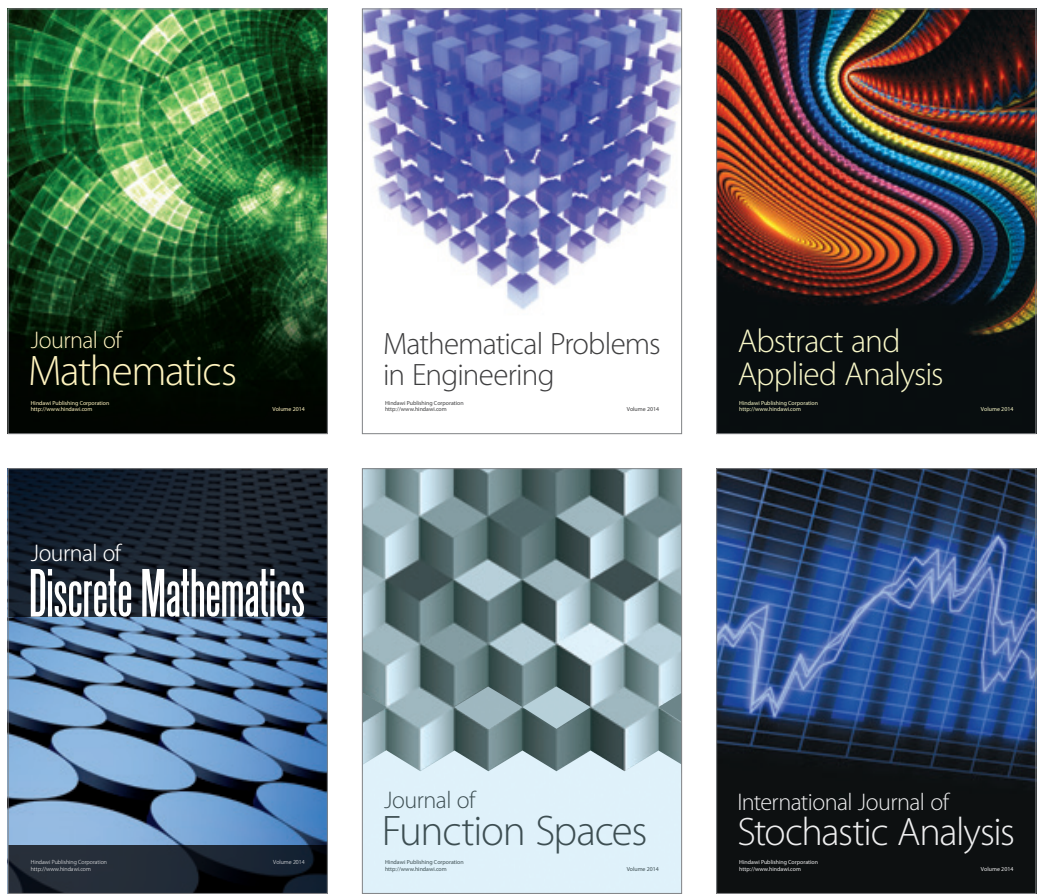

Journal of

Function Spaces

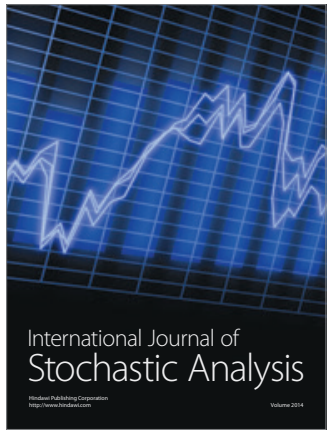

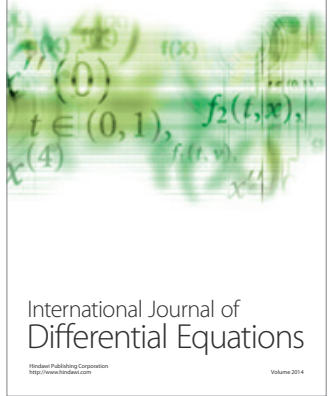
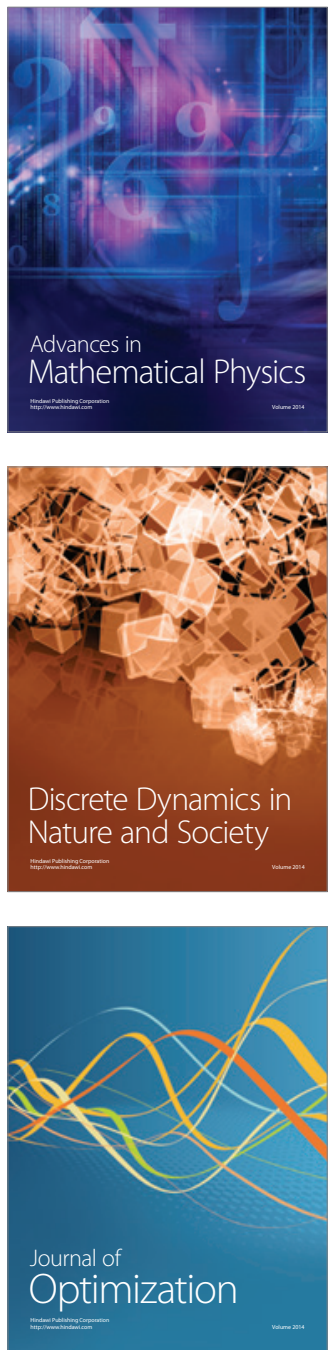